\title{
High Order Singular Rank One Perturbations of a Positive Operator
}

\author{
A. Dijksma, P. Kurasov and Yu. Shondin
}

\begin{abstract}
In this paper self-adjoint realizations in Hilbert and Pontryagin spaces of the formal expression

$$
L_{\alpha}=L+\alpha\langle\cdot, \varphi\rangle \varphi
$$

are discussed and compared. Here $L$ is a positive self-adjoint operator in a Hilbert space $\mathcal{H}$ with inner product $\langle\cdot, \cdot\rangle, \alpha$ is a real parameter, and $\varphi$ in the rank one perturbation is a singular element belonging to $\mathcal{H}_{-n} \backslash \mathcal{H}_{-n+1}$ with $n \geq 3$, where $\left\{\mathcal{H}_{s}\right\}_{s=-\infty}^{\infty}$ is the scale of Hilbert spaces associated with $L$ in $\mathcal{H}$.

Mathematics Subject Classification (2000). Primary: 47B25, 47B50; Secondary: 81Q10.

Keywords. Hilbert space, scale of Hilbert spaces, Pontryagin space, defect function, $Q$-function, symmetric operator, self-adjoint extension, rank one perturbation, Gelfand triple.
\end{abstract}

\section{Introduction}

\subsection{The singular perturbation problem}

Let $\mathcal{H}$ be a Hilbert space with inner product $\langle\cdot, \cdot\rangle$ and let $L$ be a positive selfadjoint operator in $\mathcal{H}$. Denote by $\left(\mathcal{H}_{n}\right)_{n=-\infty}^{\infty}$ the scale of Hilbert spaces associated with $L$ and $\mathcal{H}: \mathcal{H}_{0}=\mathcal{H}$, for $n>0, \mathcal{H}_{n}$ is the Hilbert space $\operatorname{dom} L^{n / 2}$ equipped with norm

$$
\|f\|_{n}=\left\|(L+1)^{n / 2} f\right\|,
$$

and for $n<0, \mathcal{H}_{n}$ is the completion of $\mathcal{H}$ with respect to the norm (1.1). In a natural way $\mathcal{H}_{n}$ and $\mathcal{H}_{-n}$ are duals and the inner product can be generalized to a pairing $\langle f, g\rangle$ between the spaces $\mathcal{H}_{n}$ and $\mathcal{H}_{-n}$ :

$$
|\langle f, g\rangle| \leq\|f\|_{n}\|g\|_{-n}, \quad f \in \mathcal{H}_{n}, g \in \mathcal{H}_{-n},
$$

The research for this paper was supported by the Netherlands Organization of Scientific Research NWO (grant 047-008-008) and by the Royal Swedish Academy of Sciences. 
and $\langle g, f\rangle=\langle f, g\rangle^{*}$. For $\pm n, m=1,2, \ldots$, the operator $(L+1)^{-m / 2}$ is an isometry from $\mathcal{H}_{n}$ to $\mathcal{H}_{n+m}$. Finally, $\mathcal{H}_{n} \hookrightarrow \mathcal{H}_{m}, n>m, n, m \in \mathbb{Z}$, and the inclusion map is contractive and has a dense range. For more details, see, for example, [33, 2]. Later we redefine the inner product on one of the scale spaces, see (2.1).

In this paper we consider the expression

$$
L_{\alpha}=L+\alpha\langle\cdot, \varphi\rangle \varphi
$$

It is called a rank one perturbation of $L$ with coupling parameter $\alpha$, which is a real number, and generalized element $\varphi$, which is an element of the space $\mathcal{H}_{-n} \backslash \mathcal{H}_{-n+1}$, $n=0,1,2, \ldots ;$ the perturbation $\alpha\langle\cdot, \varphi\rangle \varphi$ is also called an interaction. If $n=0$, or more generally, if $\varphi \in \mathcal{H}_{0}$, the perturbation (1.2) is called regular, otherwise it is called singular. The cases $n=0,1$, and 2 are well known; we give a short overview below. In this paper we focus on high order singular perturbations, that is, on perturbations with $n \geq 3$. Then $L_{\alpha}$ is just a formal expression on $\mathcal{H}_{0}$ and the aim of this paper is to present for this expression (one-parameter families of) self-adjoint realizations, that is, operators or relations, in a Hilbert or Pontryagin space. For the theory of operators on spaces with an indefinite, we refer to $[4,8,18]$. We thank the referee for his useful remarks.

\subsection{The extension method}

Self-adjoint realizations of $L_{\alpha}$ for $n \geq 3$ can be obtained by a procedure as used in, for example, spectral theory of formally symmetric differential expressions; see, for instance, [11]. If $\ell$ is such an expression, one associates with $\ell$ a minimal and a maximal realization in a suitable inner product space of functions. The minimal realization is a closed symmetric operator whose adjoint is the maximal realization. The self-adjoint realizations of $\ell$ in the space (assuming they exist) are self-adjoint extensions of the minimal realization and hence restrictions of the maximal one: these restrictions are the self-adjoint boundary conditions. To get good eigenfunction expansion results for the self-adjoint realizations, the inner product space and the maximal realization should be chosen such that the domain of the latter contains sufficiently many eigenfunctions, that is, solutions of the equation $\ell y=z y$, and such that these eigenfunctions form a dense set. The description of all selfadjoint extensions is part of extension theory which concerns defect functions, $Q$-functions, Krein's resolvent parametrization formula, etc. We shall use these notions also in the setting of Pontryagin spaces; see [21] and also [12].

\subsection{The $A$ - and $B$-models}

In this paper we associate with the singular perturbation (1.2), in a similar way, two suitable inner product spaces and two maximal operators whose domains contain the solutions of $\left(L_{\alpha}-z\right) f=0$, that is, the elements

$$
\frac{1}{L-z} \varphi, \quad z \in \rho(L)
$$

This gives rise to two kinds of self-adjoint realizations and we explain what they have in common and where they differ. Roughly speaking, what they have in 
common is that they are constructed starting from the same space $\mathbf{H}$ and the same closed maximal operator $\mathbf{L}_{\max }$ in this space, and where they differ is that subsequently in each case the space $\mathbf{H}$ is provided with a different new inner product. In the historically first approach to the singular perturbation problem the inner product is indefinite and leads to a one-parameter family of self-adjoint realizations of $L_{\alpha}$ in a Pontryagin space. We call this family the $B$-model after F. Berezin [6] who first introduced such models. The $B$-models were used and further developed in $[31,32,17,30,19,20,10,13,14,15]$. The other one-parameter family of self-adjoint realizations, which we call the $A$-model, was proposed recently in $[27,28,24,25]$. In the case $n=3$ it was shown in [27] that a nontrivial realization of (1.2) exists in a Hilbert space. In this paper we show that this is also true for higher singular perturbations.

The relation between the operators in the $A$ - and $B$-models can be described by the following commutative diagram:

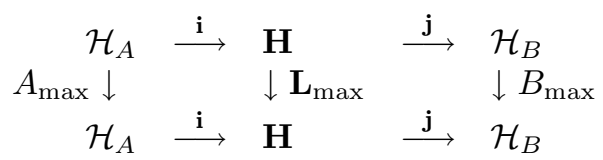

In the middle are the space and the maximal operator we start with. Using the embeddings $\mathbf{i}$ and $\mathbf{j}$ the operator $\mathbf{L}_{\max }$ can be pulled backward and pushed forward to the operator $A_{\max }$ in the space $\mathcal{H}_{A}$ in the $A$-model on the lefthand side and to the operator $B_{\max }$ in the space $\mathcal{H}_{B}$ in the $B$-model on the righthand side, respectively. The space $\mathcal{H}_{A}$ can be a Hilbert space or a Pontryagin space, depending on other parameters that come into play. The embedding $\mathbf{i}$ is an isomorphism and hence the operator $A_{\max }$ is a closed operator in $\mathcal{H}_{A}$. The inner product space $\mathcal{H}_{B}$ is a pre-Pontryagin space. The embedding $\mathbf{j}$ is continuous but not boundedly invertible, and the operator $B_{\max }$ is not closed. It turns out that its closure in the completion of the space $\mathcal{H}_{B}$ is a linear relation. In this paper we focus on the operators in the space $\mathcal{H}_{B}$ rather than on the closures of these operators in its completion. This makes the comparison between the two models more transparent. To make sure that the adjoints of the maximal operators are symmetric, additional restrictions on the parameters in the inner products have to be imposed. The selfadjoint restrictions of the maximal operators are the self-adjoint realizations of the singular perturbation.

\subsection{The regularization method}

Another way to generate the same self-adjoint realizations for $L_{\alpha}$ is to apply a regularization method to the formal expression $\left\langle\frac{1}{L-z} \varphi, \varphi\right\rangle$ when $\varphi \in \mathcal{H}_{-n} \backslash \mathcal{H}_{-n+1}$, $n \geq 3$. This procedure is analogous to the regularization of dispersion integrals in quantum field theory, see, for example, [9, 26]: Let $a_{1}, a_{2}, \ldots, a_{n-1}$ be $n-1$ positive real numbers, and set $b_{0}(z)=1$ and

$$
b_{j}(z)=\left(z+a_{1}\right)\left(z+a_{2}\right) \cdots\left(z+a_{j}\right), \quad j=1,2, \ldots, n-1 .
$$


By the resolvent identity for $L$ we have

$$
\frac{1}{L-z}=\frac{1}{b_{1}(L)}+\frac{b_{1}(z)}{b_{2}(L)}+\cdots+\frac{b_{n-2}(z)}{b_{n-1}(L)}+\frac{b_{n-1}(z)}{b_{n-1}(L)} \frac{1}{L-z},
$$

which leads to the formal identity

$$
\begin{aligned}
& \left\langle\frac{1}{L-z} \varphi, \varphi\right\rangle \stackrel{\text { formal }}{=}\left\langle\frac{1}{b_{1}(L)} \varphi, \varphi\right\rangle+b_{1}(z)\left\langle\frac{1}{b_{2}(L)} \varphi, \varphi\right\rangle \\
& \quad+\cdots+b_{n-2}(z)\left\langle\frac{1}{b_{n-1}(L)} \varphi, \varphi\right\rangle+b_{n-1}(z)\left\langle\frac{1}{b_{n-1}(L)} \frac{1}{L-z} \varphi, \varphi\right\rangle
\end{aligned}
$$

If we substitute real numbers $c_{j-1}$ for the formal pairings

$$
\left\langle\frac{1}{b_{j}(L)} \varphi, \varphi\right\rangle, \quad j=1,2, \ldots, n-1,
$$

the righthand side becomes a well defined function which we denote by $Q(z)$ :

$$
Q(z)=b_{n-1}(z)\left\langle\frac{1}{L-z} \varphi, \frac{1}{b_{n-1}(L)} \varphi\right\rangle+p_{n-2}(z)
$$

with

$$
p_{n-2}(z)=c_{0}+c_{1} b_{1}(z)+\cdots+c_{n-2} b_{n-2}(z) .
$$

The function $Q(z)$ is called a regularization of $\left\langle\frac{1}{L-z} \varphi, \varphi\right\rangle$ for $\varphi \in \mathcal{H}_{-n} \backslash \mathcal{H}_{-n+1}$. Clearly, it is not unique. The family of regularizations $Q(z)$ with fixed positive real numbers $a_{1}, a_{2}, \ldots, a_{n-1}$ can be parametrized by the polynomials $p_{n-2}(z)$ with real coefficients and of degree at most $n-2$. It can be shown that a change in the numbers $a_{1}, a_{2}, \ldots, a_{n-1}$ corresponds to a change in the polynomial $p_{n-2}(z)$. For this reason these numbers are called the normalization points. The functions $Q(z)$ are generalized Nevanlinna functions with $[(n-1) / 2]$ negative squares. The class $N_{\kappa}$ of generalized Nevanlinna functions with $\kappa$ negative squares was introduced by M.G. Krein and H. Langer in [21]. Each function from $N_{\kappa}$ is the $Q$-function of a symmetric operator with defect indices $(1,1)$ and a self-adjoint extension in a Pontryagin space with negative index $\kappa$. The one-parameter family of self-adjoint extensions of this symmetric operator is interpreted as the family of realizations of $L_{\alpha}$. This approach leads to the $B$-model. In a similar way a family of self-adjoint realizations of $L_{\alpha}$ is obtained in the $A$-model. The Hilbert space structure from the $A$-model can be explained in part by writing $Q(z)$ as

$$
Q(z)=b_{n-2}(z)\left(Q_{0}(z)+r(z)\right),
$$

where $r(z)=\left(p_{n-2}(z)-c_{n-2} b_{n-2}(z)\right) / b_{n-2}(z)$ is a generalized Nevanlinna function, but

$$
Q_{0}(z)=\left(z+a_{n-1}\right)\left\langle\frac{1}{L-z} \varphi, \frac{1}{b_{n-1}(L)} \varphi\right\rangle+c_{n-2}
$$

is a Nevanlinna function and hence the $Q$-function of a symmetric operator with defect indices $(1,1)$ and a self-adjoint extension in a Hilbert space. 


\subsection{The contents of the paper}

Besides this introduction, there are 5 sections. In Section 2 we define the space $\mathbf{H}$ and the operator $\mathbf{L}_{\max }$ from which the models are constructed. The $A$-model and $B$-model are defined in Sections 3 and 4 respectively. The one-parameter families $A_{\theta}$ and $B_{\theta}$ of self-adjoint operators are the realizations of the singular perturbation $L_{\alpha}$ (1.2). The two models are compared in Section 5, and in Section 6 we provide some examples.

1.6. The cases $n=0,1,2$

We show the differences between the perturbations for $0 \leq n \leq 2$ and $n \geq 3$ and their analogies by briefly recalling some of the results for the smaller values of $n$; see $[2]$.

(i) If $n=0$ or, more generally, $\varphi \in \mathcal{H}_{0}$, the interaction

$$
\alpha\langle\cdot, \varphi\rangle \varphi
$$

defines a bounded self-adjoint operator on $\mathcal{H}$ and $L_{\alpha}$ is a self-adjoint operator in $\mathcal{H}$ with $\operatorname{dom} L_{\alpha}=\operatorname{dom} L$. The resolvent of $L_{\alpha}$ is given by

$$
\frac{1}{L_{\alpha}-z}=\frac{1}{L-z}-\frac{1}{Q_{1}(z)+\frac{1}{\alpha}}\left\langle\cdot, \frac{1}{L-z^{*}} \varphi\right\rangle \frac{1}{L-z} \varphi,
$$

where

$$
Q_{1}(z)=\left\langle\frac{1}{L-z} \varphi, \varphi\right\rangle
$$

For $\alpha=0$ the second term on the righthand side of (1.6) should be deleted: $L_{0}=L$.

(ii) If $n=1$, the perturbation (1.5) is relatively form bounded with respect to the sesquilinear form of the operator $L$ and the perturbed operator can be determined using the form perturbation technique. Its resolvent is also given by (1.6). The main difference with the case $n=0$ is that the domain of the perturbed operator does not coincide with the domain of the original operator in general, but the perturbed operator is uniquely defined as a self-adjoint operator in the original Hilbert space $\mathcal{H}$; see $[33,2]$. Another way to obtain this operator is by considering the restriction

$$
L_{\text {min }}=\left.L\right|_{\left\{u \in \mathcal{H}_{n} \cap \operatorname{dom} L \mid\langle u, \varphi\rangle=0\right\}}
$$

with $n=1$ which is a symmetric operator in $\mathcal{H}$ with defect indices $(1,1)$. A theorem of Krein states that the resolvent formula

$$
\frac{1}{H_{\tau}-z}=\frac{1}{L-z}-\frac{1}{Q_{1}(z)+\tau}\left\langle\cdot, \frac{1}{L-z^{*}} \varphi\right\rangle \frac{1}{L-z} \varphi
$$

gives a one-to-one correspondence between all self-adjoint extensions $H_{\tau}$ of $L_{\text {min }}$ in $\mathcal{H}$ and all $\tau \in \mathbb{R} \cup\{\infty\}$. In this case we have $H_{\tau}=L_{\alpha}$ for $\tau=1 / \alpha$. Note that $Q_{1}(z)$ is a Nevanlinna function.

(iii) In the case $n=2$ the perturbation (1.5) is not relatively form bounded and only extension theory can be applied. The operator $L_{\min }$ in (1.7) with $n=2$ is 
still symmetric with defect indices $(1,1)$. But the perturbed operator is no longer uniquely defined. It is now interpreted as one of the self-adjoint extensions of $L_{\text {min }}$. These extensions can be parametrized by one real parameter $\gamma \in \mathbb{R} \cup\{\infty\}$ as follows:

$$
\begin{gathered}
\frac{1}{L^{\gamma}-z}=\frac{1}{L-z}-\frac{1}{Q_{2}(z)+\gamma}\left\langle\cdot, \frac{1}{L-z^{*}} \varphi\right\rangle \frac{1}{L-z} \varphi \\
Q_{2}(z)=\left\langle\frac{z+a_{1}}{(L-z)\left(L+a_{1}\right)} \varphi, \varphi\right\rangle .
\end{gathered}
$$

Here $a_{1}>0$ is a fixed real number, which plays no essential role: changing $a_{1}$ corresponds to changing the parameter $\gamma$. The relation between the parameter $\gamma$ in $L^{\gamma}$ and the coupling parameter $\alpha$ in $L_{\alpha}$ cannot be established without additional assumptions like homogeneity of the original operator $L$ and the interaction determined by $\varphi$. Note that $Q_{2}(z)$ is a Nevanlina function and a regularization of the expression $\left\langle\frac{1}{L-z)} \varphi, \varphi\right\rangle$.

(iv) If $n \geq 3$ the perturbation $L_{\alpha}$ in (1.5) cannot be treated as in the cases $n=0,1$, and 2 , because then $L_{\text {min }}$ in (1.7) is essentially self-adjoint in $\mathcal{H}$, that is, $L_{\text {min }}$ is not closed and its closure is a self-adjoint operator in $\mathcal{H}$. One needs to resort to other methods such as the ones introduced above.

\section{An intermediate space and a maximal operator.}

From now on, unless specified otherwise, we assume that the interaction $\varphi$ belongs to $\mathcal{H}_{-n} \backslash \mathcal{H}_{-n+1}$ with $n \geq 3$. We choose $n-1$ positive real normalization points $a_{1}, a_{2}, \ldots, a_{n-1}$ and associate with them the polynomials $b_{0}(z)=1$ and

$$
b_{j}(z)=\left(z+a_{1}\right)\left(z+a_{2}\right) \cdots\left(z+a_{j}\right), \quad j=1,2, \ldots n-1,
$$

and the singular elements

$$
\varphi_{j}=\frac{1}{b_{j}(L)} \varphi \in \mathcal{H}_{-n+2 j} \backslash \mathcal{H}_{-n+2 j+1}, \quad j=0,1, \ldots, n-1 .
$$

In the sequel we assume that the space $\mathcal{H}_{n-2}$ is endowed with the new inner product

$$
\langle u, v\rangle_{n-2}=\left\langle b_{n-2}(L) u, v\right\rangle, \quad u, v \in \mathcal{H}_{n-2} .
$$

2.1. The space $H$ and the operator $L_{\max }$

Our first choice for a minimal operator associated with the singular perturbation (1.2) is the operator $L_{\text {min }}$ in the space $\mathcal{H}_{n-2}$ :

$$
L_{\text {min }}=\left\{\{f, L f\} \mid f \in \mathcal{H}_{n},\langle f, \varphi\rangle=0\right\} .
$$

We frequently identify an operator with its graph to expedite the presentation. For graph notation and linear relations see for example [1, Section 51], [4, Section 2.1], and [16]. A natural candidate for the maximal operator is the adjoint $L_{\max }=L_{\min }^{\dagger}$ of $L_{\text {min }}$ relative to the Gelfand triple $\mathcal{H}_{n-2} \hookrightarrow H \hookrightarrow \mathcal{H}_{-n+2}$. Recall from, for 
example, [5] that if $\mathcal{K} \hookrightarrow \mathcal{H} \hookrightarrow \mathcal{K}^{\prime}$ is a Gelfand triple and $B$ is a densely defined operator in $\mathcal{K}$ then the adjoint of $B$ relative to the Gelfand triple is the operator in $\mathcal{K}^{\prime}$ defined by

$$
B^{\dagger}=\{\{f, g\} \mid\langle f, B u\rangle=\langle g, u\rangle, u \in \operatorname{dom} B\} .
$$

Here the scalar product in the definition should be understood as the pairing between $\mathcal{K}$ and $\mathcal{K}^{\prime}$ induced by the inner product $\langle\cdot, \cdot\rangle$ of $\mathcal{H}$. The adjoint operator just defined coincides with the standard adjoint operator in the case $\mathcal{K}=\mathcal{H}=\mathcal{K}^{\prime}$.

Theorem 2.1. The adjoint of $L_{\min }$ in $\mathcal{H}_{n-2}$ relative to the Gelfand triple $\mathcal{H}_{n-2} \hookrightarrow$ $H \hookrightarrow \mathcal{H}_{-n+2}$ is the operator in $\mathcal{H}_{-n+2}$ given by

$$
\left.L_{\max }=\left\{f+f_{1} \varphi_{1}, L f-a_{1} f_{1} \varphi_{1}\right\} \mid f \in \mathcal{H}_{-n+4}, f_{1} \in \mathbb{C}\right\} .
$$

Proof. The operator $L_{\text {min }}$ in $\mathcal{H}_{n-2}$ is densely defined, so the operator $L_{\max }=L_{\text {min }}^{\dagger}$ is well defined. If $\widetilde{f}, \widetilde{g} \in \mathcal{H}_{-n+2}$, then $\{\widetilde{f}, \widetilde{g}\} \in L_{\max }$ if and only if

$$
0=\langle\widetilde{f}, L u\rangle-\langle\widetilde{g}, u\rangle=\langle L \widetilde{f}-\widetilde{g}, u\rangle, \quad u \in \operatorname{dom} L_{\min },
$$

and hence if and only if $L \widetilde{f}-\widetilde{g}=f_{1} \varphi$ for some $f_{1} \in \mathbb{C}$. It follows that

$$
\widetilde{f}=\left(L+a_{1}\right)^{-1}\left(\widetilde{g}+a_{1} \widetilde{f}\right)+f_{1} \varphi_{1}=f+f_{1} \varphi_{1},
$$

where $f=\left(L+a_{1}\right)^{-1}\left(\widetilde{g}+a_{1} \widetilde{f}\right) \in \mathcal{H}_{-n+4}$, and

$$
\widetilde{g}=L \widetilde{f}-f_{1} \varphi=L f+f_{1}\left(L \varphi_{1}-\varphi\right)=L f-a_{1} f_{1} \varphi_{1} .
$$

The space $\mathcal{H}_{-n+2}$ in which the maximal operator is defined is too large for our considerations. It is sufficient that it contains the functions $\frac{1}{L-z} \varphi, z \in \rho(L)$, and the space $\mathcal{H}_{n-2}$ in which the minimal operator acts. In view of the resolvent formula (1.4) we consider the linear space

$$
\mathbf{H}=\mathcal{H}_{n-2} \dot{+} \operatorname{span}\left\{\varphi_{1}, \varphi_{2}, \ldots, \varphi_{n-2}\right\}
$$

equipped with the inner product

$$
\left\langle u+\sum_{j=1}^{n-2} \varphi_{j} u_{j}, v+\sum_{j=1}^{n-2} \varphi_{j} v_{j}\right\rangle_{\mathbf{H}}=\langle u, v\rangle_{n-2}+\sum_{j=1}^{n-2} v_{j}^{*} u_{j},
$$

where $u, v \in \mathcal{H}_{n-2}$ and $u_{j}, v_{j} \in \mathbb{C}$.It is contained in $\mathcal{H}_{-n+2}$ and only a finite dimensional extension of $\mathcal{H}_{n-2}$. By the resolvent formula (1.4) the space $\mathbf{H}$ does not depend on the choice of the normalization points $a_{j}$. The space is large enough to contain the ranges of the bounded operators

$$
R(z)=\frac{1}{L-z}-\frac{1}{Q(z)}\left\langle\cdot \frac{1}{L-z^{*}} \varphi\right\rangle \frac{1}{L-z} \varphi, \quad z \in \rho(L), Q(z) \neq 0,
$$

mapping $\mathcal{H}_{n-2}$ to $\mathcal{H}_{-n+2}$. Indeed,

$$
\operatorname{ran} R(z) \subset \mathcal{H}_{n} \dot{+} \operatorname{span}\left\{\varphi_{1}, \varphi_{2}, \ldots, \varphi_{n-2}, \varphi_{n-1}\right\}=: \mathbf{D}
$$

and $\mathbf{D} \subset \mathbf{H}$. Note that $R(z)$ is defined by a formula similar to the righthand side of Krein's formula (1.8) to which we shall refer again later. 
Evidently, the domain of the operator $L_{\max }$ contains the space $\mathbf{H}$. But the range of the restriction of $L_{\max }$ to $\mathbf{H}$ is not contained in $\mathbf{H}$. A suitable restriction of $L_{\max }$ which has this property is the restriction to the space $\mathbf{D}$ just defined. We denote this operator in $\mathbf{H}$ by $\mathbf{L}_{\max }$ : $\operatorname{dom} \mathbf{L}_{\max }=\mathbf{D}$ and for elements in $\mathbf{D}$ we have

$\mathbf{L}_{\max }\left(u+u_{n-1} \varphi_{n-1}+\sum_{j=1}^{n-2} u_{j} \varphi_{j}\right)=L u-a_{n-1} u_{n-1} \varphi_{n-1}+\sum_{j=1}^{n-2}\left(u_{j+1}-a_{j} u_{j}\right) \varphi_{j}$.

Note that $\mathbf{L}_{\max }$ is closed in $\mathbf{H}$.

\subsection{Vector notation}

In the sequel we shall use the following notation. Elements in $\mathbb{C}^{n-2}$ are always considered as column vectors. If $\vec{u} \in \mathbb{C}^{n-2}$, its entries are denoted by $u_{j}$ :

$$
\vec{u}=\left(\begin{array}{llll}
u_{1} & u_{2} & \cdots & u_{n-2}
\end{array}\right)^{\top}
$$

and we write $\vec{u}^{*}$ for the row vector

$$
\vec{u}^{*}=\left(\begin{array}{llll}
u_{1}^{*} & u_{2}^{*} & \cdots & u_{n-2}^{*}
\end{array}\right) .
$$

The inner product in $\mathbb{C}^{n-2}$ is given by $\langle\vec{u}, \vec{v}\rangle_{\mathbb{C}^{n-2}}=\vec{v}^{*} \vec{u}$. By $\vec{e}_{1}, \vec{e}_{2}, \ldots, \vec{e}_{n-2}$ we denote the standard basis in $\mathbb{C}^{n-2}$. Thus, for example, we have $\vec{e}_{j}^{*} \vec{u}=u_{j}$. The vector $\vec{\varphi}$ stands for the row vector

$$
\vec{\varphi}=\left(\begin{array}{llll}
\varphi_{1} & \varphi_{2} & \cdots & \varphi_{n-2}
\end{array}\right)
$$

so that

$$
\vec{\varphi} \vec{u}=\sum_{j=1}^{n-2} \varphi_{j} u_{j}, \quad L \vec{\varphi}=\left(\begin{array}{llll}
L \varphi_{1} & L \varphi_{2} & \cdots & L \varphi_{n-2}
\end{array}\right),
$$

and the inner product $(2.2)$ can be shortened to

$$
\langle u+\vec{\varphi} \vec{u}, v+\vec{\varphi} \vec{v}\rangle_{\mathbf{H}}=\langle u, v\rangle_{n-2}+\vec{v}^{*} \vec{u} .
$$

We extend the vector notation to the pairing:

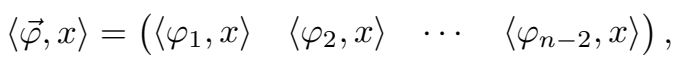

whenever the pairings on the righthand side are defined, and $\langle x, \vec{\varphi}\rangle=\langle\vec{\varphi}, x\rangle^{*}$, so that, for example,

$$
\langle\vec{\varphi} \vec{u}, x\rangle=\langle\vec{\varphi}, x\rangle \vec{u}, \quad\langle x, L \vec{\varphi} \vec{u}\rangle=\vec{u}^{*}\langle x, L \vec{\varphi}\rangle .
$$

These formulas also make sense when $\vec{u} \in \mathbb{C}^{n-2}$ is replaced by a matrix of size $(n-2) \times k$ for some $k$. 
Finally, we introduce the $(n-2) \times(n-2)$ matrix

$$
\mathfrak{M}=\left(\begin{array}{cccccccc}
-a_{1} & 1 & 0 & 0 & \ldots & 0 & 0 & 0 \\
0 & -a_{2} & 1 & 0 & \ldots & 0 & 0 & 0 \\
0 & 0 & -a_{3} & 1 & \ldots & 0 & 0 & 0 \\
0 & 0 & 0 & -a_{4} & \ldots & 0 & 0 & 0 \\
\ldots & \ldots & \ldots & \ldots & \ldots & \ldots & \ldots & \ldots \\
0 & 0 & 0 & 0 & \ldots & -a_{n-4} & 1 & 0 \\
0 & 0 & 0 & 0 & \ldots & 0 & -a_{n-3} & 1 \\
0 & 0 & 0 & 0 & \ldots & 0 & 0 & -a_{n-2}
\end{array}\right) .
$$

Then (2.3) can be written as

$$
\mathbf{L}_{\max }\left(u+u_{n-1} \varphi_{n-1}+\vec{\varphi} \vec{u}\right)=L u-a_{n-1} u_{n-1} \varphi_{n-1}+\vec{\varphi}\left(\mathfrak{M} \vec{u}+u_{n-1} \vec{e}_{n-2}\right) .
$$

This formula is the starting point for the maximal operators in the $A$ - and $B$ models, which we introduce in the next two sections.

\section{The $A$-model}

This section concerns the lefthand side of the commutative diagram (1.3) in the Introduction:

$$
\begin{array}{rll}
\mathcal{H}_{A} & \stackrel{\mathbf{i}}{\longrightarrow} & \mathbf{H} \\
A_{\max } \downarrow & & \downarrow \mathbf{L}_{\max } \\
\mathcal{H}_{A} & \stackrel{\mathbf{i}}{\longrightarrow} & \mathbf{H}
\end{array}
$$

Our aim is to construct a one-parameter family of self-adjoint operators $A_{\theta}$ acting in an inner product space $\mathcal{H}_{A}$ topologically isomorphic to $\mathbf{H}$ under the isomorphism i. The self-adjoint operators are restrictions of the operator $A_{\max }$ which is the copy of $L_{\max }$ under $\mathbf{i}$. They are the self-adjoint realizations of the singular perturbation $L_{\alpha}$ in (1.2). In general the space $\mathcal{H}_{A}$ will be a Pontryagin space, but the interesting feature of this model is that when the normalization points $a_{j}$ are mutually distinct the inner product can be chosen so that $\mathcal{H}_{A}$ is a Hilbert space.

\subsection{The space $\mathcal{H}_{A}$ and the maximal operator}

We consider the space $\mathcal{H}_{A}=\mathbb{C}^{n-2} \oplus \mathcal{H}_{n-2}$ of elements of the form

$$
U=\left(\begin{array}{l}
\vec{u} \\
u
\end{array}\right), \quad \vec{u} \in \mathbb{C}^{n-2}, u \in \mathcal{H}_{n-2},
$$

and endow it with the inner product determined by the Gram matrix

$$
G_{A}=\Gamma_{\alpha} \oplus I_{\mathcal{H}_{n-2}},
$$

that is, by the formula (see also (2.1))

$$
\langle U, V\rangle_{A}=\vec{v}^{*} \Gamma_{\alpha} \vec{u}+\left\langle b_{n-2}(L) u, v\right\rangle,
$$


where $\Gamma_{\alpha}=\left(\alpha_{j, k}\right)_{j, k=1}^{n-2}$ is a non-degenerate Hermitian $(n-2) \times(n-2)$ matrix whose entries $\alpha_{j, k}$ will be specified later. Thus $\mathcal{H}_{A}$ is a Hilbert space if $\Gamma_{\alpha}$ is positive and a Pontryagin space otherwise. The mapping i : $\mathcal{H}_{A} \rightarrow \mathbf{H}$ defined by

$$
\mathbf{i}\left(\begin{array}{l}
\vec{u} \\
u
\end{array}\right)=\vec{\varphi} \vec{u}+u
$$

is a natural topological isomorphism and we define the maximal operator $A_{\max }$ in $\mathcal{H}_{A}$ as the isomorphic copy of $\mathbf{L}_{\max }$ in $\mathbf{H}$ :

$$
\begin{aligned}
A_{\max } & =\mathbf{i}^{-1} \mathbf{L}_{\max } \mathbf{i} \\
\quad= & \left.\left\{\left(\begin{array}{c}
\vec{u} \\
u_{r}+u_{n-1} \varphi_{n-1}
\end{array}\right),\left(\begin{array}{c}
\mathfrak{M} \vec{u}+u_{n-1} \vec{e}_{n-2} \\
L u_{r}-a_{n-1} u_{n-1} \varphi_{n-1}
\end{array}\right)\right\} \mid \begin{array}{c}
\vec{u} \in \mathbb{C}^{n-2}, u_{n-1} \in \mathbb{C}, \\
u_{r} \in \mathcal{H}_{n}
\end{array}\right\} .
\end{aligned}
$$

The minimal operator is defined by $A_{\min }=A_{\max }^{*}$, the adjoint of $A_{\max }$ in $\mathcal{H}_{A}$. Since $\mathbf{L}_{\max }$ is closed, $A_{\max }$ is closed and hence $A_{\min }^{*}=A_{\max }$.

Theorem 3.1. The operator $A_{\min }$ is given by

$$
A_{\text {min }}=\left\{\left\{\left(\begin{array}{l}
\vec{u} \\
u
\end{array}\right),\left(\begin{array}{c}
\Gamma_{\alpha}^{-1} \mathfrak{M}^{*} \Gamma_{\alpha} \vec{u} \\
L u
\end{array}\right)\right\} \mid \begin{array}{c}
\vec{u} \in \mathbb{C}^{n-2}, u \in \mathcal{H}_{n}, \\
\langle u, \varphi\rangle-\vec{e}_{n-2}^{*} \Gamma_{\alpha} \vec{u}=0
\end{array}\right\}
$$

and it is symmetric if and only if $\Gamma_{\alpha}$ satisfies the relation

$$
\Gamma_{\alpha} \mathfrak{M}-\mathfrak{M}^{*} \Gamma_{\alpha}=0 .
$$

In this case the symmetric operator $A_{\min }$ is the restriction of $A_{\max }$ by two conditions:

$$
\begin{aligned}
\operatorname{dom} & A_{\min } \\
= & \left\{\left(\begin{array}{c}
\vec{u} \\
u_{r}+u_{n-1} \varphi_{n-1}
\end{array}\right) \in \operatorname{dom} A_{\max } \mid u_{n-1}=0,\left\langle u_{r}, \varphi\right\rangle-\vec{e}_{n-2}^{*} \Gamma_{\alpha} \vec{u}=0\right\} .
\end{aligned}
$$

Note that the operator $A_{\text {min }}$ is not isomorphic under $\mathbf{i}$ with the operator $L_{\text {min }}$ defined in (1.7).

Proof of Theorem 3.1. If $F, G \in \mathcal{H}_{A}$, then $\{F, G\} \in A_{\min }$ if and only if for all $\vec{u} \in \mathbb{C}^{n-2}, u_{n-2} \in \mathbb{C}$, and $u_{r} \in \mathcal{H}_{n}$ we have

$$
\begin{aligned}
0= & \left\langle F, A_{\max }\left(\begin{array}{c}
\vec{u} \\
u_{r}+u_{n-1} \varphi_{n-1}
\end{array}\right)\right\rangle_{A}-\left\langle G,\left(\begin{array}{c}
\vec{u} \\
u_{r}+u_{n-1} \varphi_{n-1}
\end{array}\right)\right\rangle_{A} \\
= & \left(\mathfrak{M} \vec{u}+u_{n-1} \vec{e}_{n-2}\right)^{*} \Gamma_{\alpha} \vec{f}-\left\langle b_{n-2}(L) f, L u_{r}-a_{n-1} u_{n-1} \varphi_{n-1}\right\rangle \\
& -\vec{u}^{*} \Gamma_{\alpha} \vec{g}-\left\langle b_{n-2}(L) g, u_{r}+u_{n-1} \varphi_{n-1}\right\rangle .
\end{aligned}
$$

Choosing $\vec{u}=0$ and $u_{n-1}=0$ we find that for all $u_{r} \in \mathcal{H}_{n}$,

$$
0=\left\langle b_{n-2}(L) f, L u_{r}\right\rangle-\left\langle b_{n-2}(L) g, u_{r}\right\rangle
$$

and hence

$$
f \in \mathcal{H}_{n}, \quad g=L f .
$$


It follows that for all $\vec{u} \in \mathbb{C}^{n-2}$ and $u_{n-1} \in \mathbb{C}$,

$$
\begin{aligned}
0 & =\left(\mathfrak{M} \vec{u}+u_{n-1} \vec{e}_{n-2}\right)^{*} \Gamma_{\alpha} \vec{f}+\left\langle f, a_{n-1} u_{n-1} \varphi_{n-1}\right\rangle-\vec{u}^{*} \Gamma_{\alpha} \vec{g}-\left\langle g, u_{n-1} \varphi_{n-1}\right\rangle \\
& =\left(\mathfrak{M} \vec{u}+u_{n-1} \vec{e}_{n-2}\right)^{*} \Gamma_{\alpha} \vec{f}-\vec{u}^{*} \Gamma_{\alpha} \vec{g}+\left\langle f, u_{n-1} \varphi_{n-2}\right\rangle,
\end{aligned}
$$

where we used that $\left(L+a_{n-1}\right) \varphi_{n-1}=\varphi_{n-2}$. Choosing $u_{n-1}=0$ we find that

$$
\vec{g}=\Gamma_{\alpha}^{-1} \mathfrak{M}^{*} \Gamma_{\alpha} \vec{f},
$$

and choosing $\vec{u}=0$ we obtain

$$
\langle f, \varphi\rangle-\vec{e}_{n-2}^{*} \Gamma_{\alpha} \vec{f}=0 .
$$

The calculations can be traced backwards to complete the proof of the representation of $A_{\min }$.

Evidently, $\operatorname{dom} A_{\min } \subset \operatorname{dom} A_{\max }$ and if $\Gamma_{\alpha}$ is a solution of (3.2) then $A_{\min } \subset$ $A_{\max }$, that is, $A_{\min }$ is symmetric. To prove the converse, assume that $A_{\min }$ is symmetric. Choose an arbitrary vector $\vec{f} \in \mathbb{C}^{n-2}$ and then choose an $f \in \mathcal{H}_{n}$ such that (3.3) holds and set $F=\left(\begin{array}{l}\vec{f} \\ f\end{array}\right)$. The symmetry implies that $A_{\min } F=A_{\max } F$ and hence

$$
\Gamma_{\alpha}^{-1} \mathfrak{M}^{*} \Gamma_{\alpha} \vec{f}=\mathfrak{M} \vec{f} .
$$

Since $\vec{f}$ is arbitrary, $\Gamma_{\alpha}$ satisfies (3.2).

The proof of the last statement is left to the reader.

The following theorem shows that if the diagonal entries of the matrix $\mathfrak{M}$ are mutually distinct, then there are many positive Hermitian solutions $\Gamma_{\alpha}$ of the equation (3.2); otherwise the Hermitian solutions are necessarily non-positive.

Theorem 3.2. (i) The equation (3.2) has a family of Hermitian solutions which can be parametrized with $n-2$ real parameters. This family contains infinitely many non-degenerate (and infinitely many degenerate) solutions.

(ii) If the normalization points $a_{j}$ are mutually distinct, then (3.2) has a family of positive Hermitian solutions which can be parametrized with $n-2$ real parameters.

(iii) If at least two of the $a_{j}$ 's are equal then every non-degenerate Hermitian solution of $(3.2)$ is indefinite.

Proof. (i) Equation (3.2) implies that all entries $\alpha_{j, k}$ of the Hermitian matrix $\Gamma_{\alpha}$ are real

$$
\alpha_{j, k}=\alpha_{k, j} \in \mathbb{R}
$$

and satisfy the following recurrence relations

$$
\begin{array}{ll}
\alpha_{j, k}=\alpha_{j-1, k+1}+\left(a_{k+1}-a_{j}\right) \alpha_{j, k+1}, & 2 \leq j \leq k \leq n-3, \\
\alpha_{1, k}=\left(a_{k+1}-a_{1}\right) \alpha_{1, k+1}, & k=1, \ldots, n-3 .
\end{array}
$$

These equations show that the last column $\vec{\gamma}$ of the matrix $\Gamma_{\alpha}$ can be chosen arbitrarily among real vectors and that the entries of the first row are determined by the value of the last entry. They allow one to calculate all entries $\alpha_{j, k}, j \leq k$. 
All entries below the diagonal are determined taking into account that $\Gamma_{\alpha}$ is symmetric. The solution $\Gamma_{\alpha}$ depends continuously on $\vec{\gamma}$ and if we take $\vec{\gamma}=\vec{e}_{1}$ then $\Gamma_{\alpha}$ is non-degenerate; hence if we consider $\vec{\gamma}$ with nonzero first entry, then for sufficiently small values of the other entries the solution will be non-degenerate also. If we choose $\gamma_{1}=0$, then the second recurrence equality in (3.4) implies that the first row of the solution is the zero vector and hence the solution has a zero determinant.

(ii) Consider the following family of upper triangular matrices $Y$

$$
Y=\operatorname{diag}\left(\xi_{1}, \xi_{2}, \ldots, \xi_{n-2}\right) X
$$

where $\xi_{j}, j=1, \ldots, n-2$, are arbitrary nonzero complex numbers and $X$ is the $(n-2) \times(n-2)$ matrix

$X=$

$$
\left(\begin{array}{ccccc}
\left(a_{n-2}-a_{1}\right) \ldots\left(a_{2}-a_{1}\right) & \left(a_{n-2}-a_{1}\right) \ldots\left(a_{3}-a_{1}\right) & \ldots & \left(a_{n-2}-a_{1}\right) & 1 \\
0 & \left(a_{n-2}-a_{2}\right) \ldots\left(a_{3}-a_{2}\right) & \ldots & \left(a_{n-2}-a_{2}\right) & 1 \\
\vdots & \vdots & \ddots & \vdots & \vdots \\
0 & 0 & \ldots & \left(a_{n-2}-a_{n-3}\right) & 1 \\
0 & 0 & \ldots & 0 & 1
\end{array}\right) .
$$

Then the matrix $\Gamma_{\alpha}=Y^{*} Y$ satisfies equation (3.2). Indeed, we have that

$$
Y \mathfrak{M}=-\operatorname{diag}\left(a_{1}, a_{2}, \ldots, a_{n-2}\right) Y,
$$

and this implies

$$
\begin{aligned}
Y^{*} Y \mathfrak{M} & =-Y^{*} \operatorname{diag}\left(a_{1}, a_{2}, \ldots, a_{n-2}\right) Y=-\left(\operatorname{diag}\left(a_{1}, a_{2}, \ldots, a_{n-2}\right) Y\right)^{*} Y \\
& =(Y \mathfrak{M})^{*} Y=\mathfrak{M}^{*} Y^{*} Y .
\end{aligned}
$$

The last column of the matrix $\Gamma_{\alpha}=Y^{*} Y$ is equal to

$$
X^{*}\left(\begin{array}{c}
\left|\xi_{1}\right|^{2} \\
\left|\xi_{2}\right|^{2} \\
\vdots \\
\left|\xi_{n-2}\right|^{2}
\end{array}\right) X,
$$

and this implies that the family of matrices just constructed is described by $n-2$ independent (positive) real parameters $\left|\xi_{j}\right|^{2}$.

(iii) Consider now the case when at least two of the parameters $a_{j}$, say $a_{1}$ and $a_{2}$, coincide. Then the second recurrence relation in (3.4) implies that $\alpha_{11}=0$ and the matrix $\Gamma_{\alpha}$ has at least one negative eigenvalue.

By way of example, suppose that all numbers $a_{j}$ are equal, say $a_{j}=a$. Then the recurrence relations (3.4) imply that the matrix $\Gamma_{\alpha}$ is a Hankel matrix $\alpha_{j, k}=\alpha_{j+k}$ 
with $\alpha_{l}=0, l=2, \ldots, n-2$, that is, $\Gamma_{\alpha}$ is an anti-triangular matrix

$$
\Gamma_{\alpha}=\left(\begin{array}{ccccc}
0 & 0 & \ldots & 0 & \alpha_{n-1} \\
0 & 0 & \ldots & \alpha_{n-1} & \alpha_{n} \\
\vdots & \vdots & \ddots & \vdots & \vdots \\
0 & \alpha_{n-1} & \ldots & \alpha_{2 n-6} & \alpha_{2 n-5} \\
\alpha_{n-1} & \alpha_{n} & \ldots & \alpha_{2 n-5} & \alpha_{2 n-4}
\end{array}\right)
$$

The number of negative eigenvalues of this matrix is equal to

$$
\begin{cases}\frac{n-2}{2}, & \text { if } n \text { is even, } \\ \frac{n-1}{2}, & \text { if } n \text { is odd and } \alpha_{n-1}>0 \\ \frac{n+1}{2}, & \text { if } n \text { is odd and } \alpha_{n-1}<0\end{cases}
$$

\subsection{The self-adjoint realizations $A_{\theta}$}

From now on we assume that $\Gamma_{\alpha}$ is an invertible Hermitian solution of (3.2). Then $A_{\text {min }}$ is a symmetric operator. Its defect indices are $(1,1)$ and a defect element for $A_{\text {min }}$ at $z \in \rho(L)$ is given by

$$
\Phi_{A}(z)=\frac{1}{b_{n-2}(z)}\left(\begin{array}{c}
\vec{b}(z) \\
\frac{1}{L-z} \frac{b_{n-2}(z)}{b_{n-2}(L)} \varphi
\end{array}\right), \quad \vec{b}(z)=\left(\begin{array}{c}
1 \\
b_{1}(z) \\
\vdots \\
b_{n-3}(z)
\end{array}\right)
$$

Indeed, writing $\Phi_{A}(z)$ as

$$
\Phi_{A}(z)=\left(\begin{array}{c}
\frac{1}{b_{n-2}(z)} \vec{b}(z) \\
u+\varphi_{n-1}
\end{array}\right), \quad u=\frac{z+a_{n-1}}{L-z} \varphi_{n-1} \in \mathcal{H}_{n}
$$

and using the identity

$$
(\mathfrak{M}-z) \vec{b}(z)=-b_{n-2}(z) \vec{e}_{n-2},
$$

we find that $\left(A_{\max }-z\right) \Phi_{A}(z)=0$. By (1.4) with $n$ replaced by $n-1$, the element $\frac{1}{L-z} \varphi$ belongs to $\mathbf{H}$ and we have

$$
\mathbf{i} \Phi_{A}(z)=\frac{1}{b_{n-2}(z)} \frac{1}{L-z} \varphi .
$$

Among all self-adjoint extensions of $A_{\text {min }}$ one resembles the original operator $L$, namely

$$
A_{0}=\mathfrak{M} \oplus L=\left\{\left\{\left(\begin{array}{l}
\vec{u} \\
u
\end{array}\right),\left(\begin{array}{l}
\mathfrak{A} \vec{u} \\
L u
\end{array}\right)\right\} \mid u \in \mathcal{H}_{n}, \vec{u} \in \mathbf{C}^{n-2}\right\},
$$

where $L$ is the self-adjoint operator on the space $\mathcal{H}_{n-2}$ with $\operatorname{dom} L=\mathcal{H}_{n}$. The operator $A_{\min }$ can be described as the restriction of the operator $A_{0}$ to the set of functions $U \in \operatorname{dom} A_{0}$ satisfying the condition

$$
\left\langle\left(A_{0}+a_{n-1}\right) U, \Phi_{A}\left(-a_{n-1}\right)\right\rangle_{A}=0 .
$$


To see this it suffices to show that this condition is equivalent to the condition

$$
\langle u, \varphi\rangle-\vec{e}_{n-2}^{*} \Gamma_{\alpha} \vec{u}=0
$$

appearing in the formula (3.1) for $A_{\min }$. This follows from

$$
\begin{aligned}
& \left\langle\left(\mathfrak{M}+a_{n-1}\right) U, \Phi_{A}\left(-a_{n-1}\right)\right\rangle_{A} \\
& =\left\langle\left(\begin{array}{c}
\left(\mathfrak{M}+a_{n-1}\right) \vec{u} \\
\left(L+a_{n-1}\right) u
\end{array}\right),\left(\frac{1}{b_{n-2}\left(-a_{n-1}\right)} \vec{b}\left(-a_{n-1}\right)\right)\right\rangle_{A} \\
& =\frac{1}{\varphi_{n-1}} \vec{b}\left(-a_{n-1}\right)^{*} \Gamma_{\alpha}\left(\mathfrak{M}+a_{n-1}\right) \vec{u}+\left\langle b_{n-2}(L)\left(L+a_{n-1}\right) u, \varphi_{n-1}\right\rangle \\
& =-\vec{e}_{n-2}^{*} \Gamma_{\alpha} \vec{u}+\langle u, \varphi\rangle,
\end{aligned}
$$

where we used $\Gamma_{\alpha} \mathfrak{M}=\mathfrak{M}^{*} \Gamma_{\alpha}$ and (3.6) with $z=-a_{n-1}$.

The operator $A_{0}$ will be used to describe all self-adjoint extensions of $A_{\min }$ via Krein's resolvent formula. The defect elements $\Phi_{A}(z)$ in (3.5) at different points are related by the Hilbert identity

$$
\Phi_{A}(z)-\Phi_{A}(\zeta)=(z-\zeta) \frac{1}{A_{0}-z} \Phi_{A}(\zeta)
$$

which means that $\Phi_{A}(z)$ is a defect function associated with $A_{0}$. The $Q$-function for the operators $A_{\min }$ and $\mathbf{A}_{0}$, by definition the solution of the equation

$$
\frac{Q_{A}(z)-Q_{A}(\zeta)^{*}}{z-\zeta^{*}}=\left\langle\Phi_{A}(z), \Phi_{A}(\zeta)\right\rangle_{A},
$$

is given by

$$
\begin{aligned}
Q_{A}(z) & =\left(z+a_{n-1}\right)\left\langle\Phi_{A}(z), \Phi_{A}\left(-a_{n-1}\right)\right\rangle_{A}+c \\
& =\left\langle\frac{z+a_{n-1}}{L-z} \varphi, d \frac{1}{b_{n-1}(L)} \varphi\right\rangle+r(z)-r\left(-a_{n-1}\right)+c,
\end{aligned}
$$

where $c$ is a real constant and $r(z)$ is the rational function

$$
r(z)=\vec{e}_{n-2}^{*} \Gamma_{\alpha} \frac{1}{\mathfrak{M}-z} \vec{e}_{n-2}=-\sum_{k=1}^{n-2} \alpha_{k, n-2} \frac{b_{k-1}(z)}{b_{n-2}(z)},
$$

where to obtain the last equality we used (3.6). We normalize $Q_{A}(z)$ by the condition $Q_{A}\left(-a_{n-1}\right)=r\left(-a_{n-1}\right)$ or, equivalently, $c=r\left(-a_{n-1}\right)$, and shall use the fixed $Q$-function

$$
Q_{A}(z)=\left\langle\frac{z+a_{n-1}}{L-z} \varphi, \frac{1}{b_{n-1}(L)} \varphi\right\rangle+r(z) .
$$

Formula (3.9) implies that $r(z) \in N_{\kappa_{1}}$, where $\kappa_{1}$ is the number of negative eigenvalues of $\Gamma_{\alpha}$. Hence $\kappa_{1} \leq n-2$. The poles of $r(z)$ lie at the points $-a_{1},-a_{2}, \ldots,-a_{n-2}$ on the negative half axis and therefore outside of the spectrum of $L$. Then by [22, Satz 1.13 ] the function $Q_{A}(z)$ belongs to the class $N_{\kappa_{1}}$ because $Q_{A}(z)$ is a sum 
of a Nevanlinna function and a function from $N_{\kappa_{1}}$ whose spectra are mutually disjoint.

Using the defect function and the $Q$-function all self-adjoint extensions of $A_{\min }$ in the space $\mathcal{H}_{A}$ can be described as a one-parameter family of operators by Krein's resolvent formula. This is formulated in the first part of the next theorem. Selfadjoint extensions of $A_{\min }$ are restrictions of $A_{\max }$ and these are described in the second part of the theorem.

Theorem 3.3. (i) The resolvent relation

$$
\frac{1}{A_{\theta}-z}=\frac{1}{A_{0}-z}-\frac{1}{Q_{A}(z)+\cot \theta}\left\langle\cdot, \Phi_{A}\left(z^{*}\right)\right\rangle_{A} \Phi_{A}(z) .
$$

defines a one-to-one correspondence between all self-adjoint extensions $A_{\theta}$ of $A_{\min }$ in $\mathcal{H}_{A}$ and the numbers $\theta \in[0, \pi)$.

(ii) The self-adjoint operator $A_{\theta}$ is semi-bounded and the restriction of $A_{\max }$ by the condition

$$
U \in \operatorname{dom} A_{\max }, \quad \cos \theta u_{n-1}+\sin \theta\left(\left\langle u_{r}, \varphi\right\rangle-\vec{e}_{n-2}^{*} \Gamma_{\alpha} \vec{u}\right)=0, \theta \in[0, \pi) .
$$

Proof. The proof of statement (i) is well known; see, for instance, [1]. We prove (ii). Consider $\theta \in(0, \pi)$ and fix a point $z \in \rho(L)$ such that $Q_{A}(z)+\cot \theta \neq 0$. Let $U \in \mathcal{H}_{A}$. Then $U \in \operatorname{dom} A_{\theta}$ if and only if for some $F \in \mathcal{H}_{A}$

$$
U=\frac{1}{A_{0}-z} F-\frac{1}{Q_{A}(z)+\cot \theta}\left\langle F, \Phi_{A}\left(z^{*}\right)\right\rangle_{A} \Phi_{A}(z) .
$$

Thus $U$ can be written as

$$
U=\left(\begin{array}{l}
\vec{u} \\
u
\end{array}\right)=V+u_{n-1} \Phi\left(-a_{n-1}\right)=\left(\begin{array}{c}
\vec{v}+\frac{u_{n-1}}{b_{n-2}\left(-a_{n-1}\right)} \vec{b}\left(-a_{n-1}\right) \\
u_{r}+u_{n-1} \varphi_{n-1}
\end{array}\right)
$$

with

$$
\begin{aligned}
u_{n-1} & =-\frac{1}{Q_{A}(z)+\cot \theta}\left\langle F, \Phi_{A}\left(z^{*}\right)\right\rangle_{A} \in \mathbb{C}, \\
V & =\left(\begin{array}{c}
\vec{v} \\
u_{r}
\end{array}\right)=\frac{1}{A_{0}-z} F+u_{n-1} \frac{z+a_{n-1}}{A_{0}-z} \Phi_{A}\left(-a_{n-1}\right) \in \operatorname{dom} A_{0} ;
\end{aligned}
$$

in particular, $u_{r} \in \mathcal{H}_{n}$. Using the defining relation (3.8) for $Q_{A}(z)$ and its normalization, we obtain

$$
\left\langle\left(A_{0}-z\right) V, \Phi_{A}\left(z^{*}\right)\right\rangle_{A}=-u_{n-1}\left(r\left(-a_{n-1}\right)+\cot \theta\right) .
$$

On the other hand using (3.6), the relation between the first components of $U$ and $V$ given by (3.12) and the formula (3.9) for the function $r(z)$ we find that the 
inner product on the lefthand side is equal to

$$
\begin{aligned}
& \left\langle\left(A_{0}-z\right) V, \Phi_{A}\left(z^{*}\right)\right\rangle_{A}= \\
& =\left\langle\left(\begin{array}{c}
(\mathfrak{M}-z) \vec{v} \\
(L-z) u_{r}
\end{array}\right),\left(\begin{array}{c}
\frac{1}{b_{n-2}\left(z^{*}\right)} \vec{b}\left(z^{*}\right) \\
\frac{1}{L-z^{*}} \varphi_{n-2}
\end{array}\right)\right\rangle_{A} \\
& =\frac{1}{b_{n-2}(z)} \vec{b}\left(z^{*}\right)^{*} \Gamma_{\alpha}(\mathfrak{M}-z) \vec{v}+\left\langle u_{r}, b_{n-2}(L) \varphi_{n-2}\right\rangle \\
& =-\vec{e}_{n-2}^{*} \Gamma_{\alpha} \vec{v}+\langle v, \varphi\rangle \\
& =-\vec{e}_{n-2}^{*} \Gamma_{\alpha} \vec{u}+\frac{u_{n-1}}{b_{n-2}\left(-a_{n-1}\right)} \vec{e}_{n-2}^{*} \Gamma_{\alpha} \vec{b}\left(-a_{n-1}\right)+\left\langle u_{r}, \varphi\right\rangle \\
& =-\vec{e}_{n-2}^{*} \Gamma_{\alpha} \vec{u}+\left\langle u_{r}, \varphi\right\rangle-u_{n-1} r\left(-a_{n-1}\right) .
\end{aligned}
$$

It follows that $U$ satisfies the condition (3.11). It is easy to show that this condition determines a symmetric extension of $A_{\text {min }}$. Therefore this extension necessarily coincides with the operator $A_{\theta}$. It follows that $A_{\theta}$ is the restriction $A_{\max }$ to the set of elements in dom $A_{\max }$ which satisfy (3.11).

In the case $\theta=0$ the self-adjoint operator coincides with $A_{0}$.

Note that in terms of $A_{0}$ and $\Phi_{A}(z)$ the maximal operator $A_{\max }=A_{\min }^{*}$ can be described as the relation

$$
\begin{gathered}
A_{\max }=\left\{\left\{U+u_{n-1} \Phi_{A}\left(-a_{n-1}\right), A_{0} U-a_{n-1} u_{n-1} \Phi_{A}\left(-a_{n-1}\right)\right\} \mid\right. \\
\left.U \in \operatorname{dom} A_{0}, u_{n-1} \in \mathbb{C}\right\}
\end{gathered}
$$

and the self-adjoint operator $A_{\theta}$ as

$$
\begin{aligned}
A_{\theta}= & \left\{\left\{U+u_{n-1} \Phi_{A}\left(-a_{n-1}\right), A_{0} U-a_{n-1} u_{n-1} \Phi_{A}\left(-a_{n-1}\right)\right\} \mid U \in \operatorname{dom} A_{0},\right. \\
& \left.u_{n-1} \in \mathbb{C},\left\langle\left(A_{0}+a_{n-1}\right) U, \Phi_{A}\left(-a_{n-1}\right)\right\rangle_{A}=-\left(r\left(-a_{n-1}\right)+\cot \theta\right) u_{n-1}\right\} .
\end{aligned}
$$

The first formula follows from (3.7); the second one from arguments in the foregoing proof.

\subsection{Compressions of the resolvent of $A_{\theta}$}

The formula for the skew compression $\left.\mathbf{i} \frac{1}{A_{\theta}-z}\right|_{\mathcal{H}_{n-2}}$ of the resolvent of $A_{\theta}$ follows immediately from (3.10):

$$
\left.\mathbf{i} \frac{1}{A_{\theta}-z}\right|_{\mathcal{H}_{n-2}}=\frac{1}{L-z}-\frac{1}{Q(z)}\left\langle\cdot, \frac{1}{L-z^{*}} \varphi\right\rangle \frac{1}{L-z} \varphi
$$

where

$$
Q(z)=b_{n-2}(z)\left(Q_{A}(z)+\cot \theta\right) .
$$

The function $Q(z)$ is always a generalized Nevanlinna function, even if the parameters of the model are chosen such that $\mathcal{H}_{A}$ is a Hilbert space.

The formula for the compression of the resolvent to $\mathcal{H}_{n-2}$ is given by:

$$
\left.P_{\mathcal{H}_{n-2}} \frac{1}{A_{\theta}-z}\right|_{\mathcal{H}_{n-2}}=\frac{1}{L-z}-\frac{1}{Q_{A}(z)+\cot \theta}\left\langle\cdot, \frac{1}{L-z^{*}} \varphi_{n-2}\right\rangle_{n-2} \frac{1}{L-z} \varphi_{n-2} .
$$


This formula implies that the spectral problem for the operator $A_{\theta}$ is equivalent to the following explicit eigenvalue depending "boundary value" problem:

$$
\begin{aligned}
(L-z) u & =c \varphi_{n-1}, \quad u \in \mathcal{H}_{n}, c \in \mathbb{C}, \\
\left(z+a_{n-1}\right)\langle u, \varphi\rangle & =-c(r(z)+\cot \theta) .
\end{aligned}
$$

\section{The $B$-model}

In this section we discuss an extended form of the righthand side of the commutative diagram (1.3) in the Introduction:

$$
\begin{array}{llllll}
\mathbf{H} & \stackrel{\mathbf{j}_{1}}{\longrightarrow} & \mathcal{H}_{B} & \stackrel{\mathbf{j}_{2}}{\longrightarrow} & \widehat{\mathcal{H}}_{B} \\
\mathbf{L}_{\max } \downarrow & & \downarrow B_{\max } & & \downarrow \widehat{B}_{\max } \\
\mathbf{H} & \stackrel{\mathbf{j}_{1}}{\longrightarrow} & \mathcal{H}_{B} & \stackrel{\mathbf{j}_{2}}{\longrightarrow} & \widehat{\mathcal{H}}_{B}
\end{array}
$$

which we use to construct a one-parameter family of self-adjoint realizations, called model B. The space $\mathcal{H}_{B}$ is a pre-Pontryagin space with negative index $\kappa=[(n-$ 1)/2] obtained from $\mathbf{H}$ by equipping it with a new inner product. The mapping $\mathbf{j}_{1}$ is a bijection. The space $\widehat{\mathcal{H}}_{B}$ on the righthand side is the completion of $\mathcal{H}_{B}$ and $\mathbf{j}_{2}$ is the natural embedding. The operator $B_{\max }$ is the adjoint of a symmetric operator $B_{\min }$ and the self-adjoint extensions are considered as the self-adjoint realizations of the singular perturbation $L_{\alpha}$ in (1.2), but strictly speaking one should consider the closures of these operators in the space $\widehat{\mathcal{H}}_{B}$.

\subsection{The space $\mathcal{H}_{B}$ and the operators $B_{\min }$ and $B_{\theta}$}

We define $\mathcal{H}_{B}$ as the inner product space $\mathcal{H}_{B}=\mathbb{C}^{n-2} \dot{+} \mathcal{H}_{n-2}$ with elements of the form

and inner product

$$
U=\left(\begin{array}{l}
\vec{u} \\
u
\end{array}\right), \quad \vec{u} \in \mathbb{C}^{n-2}, u \in \mathcal{H}_{n-2},
$$

$$
\langle U, V\rangle_{B}=\vec{v}^{*} \Gamma_{\beta} \vec{u}+\langle u, \vec{\varphi} \vec{v}\rangle+\langle\vec{\varphi} \vec{u}, v\rangle+\langle u, v\rangle .
$$

The matrix $\Gamma_{\beta}=\left(\beta_{j, k}\right)_{j, k=1}^{n-2}$ is a non-degenerate Hermitian $(n-2) \times(n-2)$ matrix whose entries below the anti-diagonal are defined by

$$
\beta_{j, k}:=\left\langle\varphi_{k}, \varphi_{j}\right\rangle=\beta_{k, j}, \quad j, k=2,3, \ldots, n-2, j+k \geq n,
$$

and the other entries will be specified later. We also set

$$
\beta_{j, n-1}=\left\langle\varphi_{n-1}, \varphi_{j}\right\rangle=\beta_{n-1, j}, j=1,2, \ldots, n-2,
$$

so that by the resolvent identity we have

$$
\beta_{j, k}=\beta_{j-1, k+1}+\left(a_{k+1}-a_{j}\right) \beta_{j, k+1}, \quad j, k=2,3, \ldots, n-2, j+k \geq n .
$$

For later use we define the numbers

$$
\beta_{j}:=\beta_{1, j}+\left(a_{1}-a_{j+1}\right) \beta_{1, j+1}, \quad j=1,2, \ldots, n-2 .
$$


We restrict our considerations to the case where the entries of $\Gamma_{\beta}$ are real. The inner product space $\mathcal{H}_{B}$ is not complete and therefore a pre-Pontryagin space and the identification mapping $\mathbf{j}_{1}: \mathbf{H} \rightarrow \mathcal{H}_{B}$ defined by

$$
\mathbf{j}_{1}(u+\vec{\varphi} \vec{u})=\left(\begin{array}{l}
\vec{u} \\
u
\end{array}\right)
$$

is a continuous bijection but its inverse is not continuous. At a later stage we shall complete the space $\mathcal{H}_{B}$. The adjoint of a densely defined operator $B$ in $\mathcal{H}_{B}$ relative to the inner product of $\mathcal{H}_{B}$ will be denoted by $B^{+}$:

$$
B^{+}=\left\{\{V, W\} \mid\langle V, B U\rangle_{B}-\langle W, U\rangle_{B}=0, U \in \operatorname{dom} B\right\} .
$$

The maximal operator $B_{\max }$ is defined by

$$
\begin{aligned}
& B_{\max }=\mathbf{j}_{1} \mathbf{L}_{\max } \mathbf{j}_{1}^{-1} \\
& \quad=\left\{\left\{\left(\begin{array}{c}
\vec{u} \\
u_{r}+u_{n-1} \varphi_{n-1}
\end{array}\right),\left(\begin{array}{c}
\mathfrak{M} \vec{u}+u_{n-1} \vec{e}_{n-2} \\
L u_{r}-a_{n-1} u_{n-1} \varphi_{n-1}
\end{array}\right)\right\} \mid \begin{array}{c}
\vec{u} \in \mathbb{C}^{n-2}, u_{n-1} \in \mathbb{C}, \\
u_{r} \in \mathcal{H}_{n}
\end{array}\right\} .
\end{aligned}
$$

The operator $B_{\max }$ is densely defined. In fact, the set $\{0\} \oplus \mathcal{H}_{n}$ contained in its domain is already dense in $\mathcal{H}_{B}$ : If $V \in \mathcal{H}_{B}$ is orthogonal to all elements $U \in$ $\{0\} \oplus \mathcal{H}_{n}$, that is,

$$
0=\langle V, U\rangle_{B}=\langle\vec{\varphi} \vec{v}+v, u\rangle, \quad u \in \mathcal{H}_{n},
$$

then $\vec{\varphi} \vec{v}+v=0$ and therefore, since the elements $\varphi_{1}, \ldots, \varphi_{n-2}$ are linearly independent modulo $\mathcal{H}_{n-2}$, we have $\vec{v}=0$ and $v=0$, so $V=0$.

Theorem 4.1. The operator $B_{\max }^{+}$is the restriction of $B_{\max }$ to all elements

$$
U=\left(\begin{array}{c}
\vec{u} \\
u_{r}+u_{n-1} \varphi_{n-1}
\end{array}\right) \in \operatorname{dom} B_{\max }
$$

which satisfy the three conditions

$$
u_{1}=0, \quad P\left(\Gamma_{\beta} \mathfrak{M}-\mathfrak{M}^{*} \Gamma_{\beta}\right) P \vec{u}=0, \quad F_{\beta}(U)=0 .
$$

Here $P$ is the orthogonal projection onto the subspace $\left\{\vec{e}_{1}\right\}^{\perp}$ of $\mathbb{C}^{n-2}$ and with $\beta_{j}$ as in (4.4)

$$
F_{\beta}(U)=\sum_{j=1}^{n-2} \beta_{j} u_{j+1}+\left\langle u_{r}, \varphi\right\rangle .
$$

Moreover, $\left(B_{\max }^{+}\right)^{+}=B_{\max }$ and $B_{\max }^{+}$is a densely defined symmetric operator.

Proof. We have $\{X, Y\} \in B_{\max }^{+}$if and only if for all $\vec{u} \in \mathbb{C}^{n-2}, u_{n-1} \in \mathbb{C}$ and $u_{r} \in \mathcal{H}_{n}$,

$$
\left\langle\left(\begin{array}{l}
\vec{x} \\
x
\end{array}\right),\left(\begin{array}{c}
\mathfrak{M} \vec{u}+u_{n-1} \vec{e}_{n-2} \\
L u_{r}-a_{n-1} u_{n-1} \varphi_{n-1}
\end{array}\right)\right\rangle_{B}-\left\langle\left(\begin{array}{l}
\vec{y} \\
y
\end{array}\right),\left(\begin{array}{c}
\vec{u} \\
u_{r}+u_{n-1} \varphi_{n-1}
\end{array}\right)\right\rangle_{B}=0
$$


that is, if and only if

$$
\begin{aligned}
& 0=L x-y+L \vec{\varphi} \vec{x}-\varphi \vec{y} \\
& 0=\mathfrak{M}^{*} \Gamma_{\beta} \vec{x}+\langle x, \vec{\varphi} \mathfrak{M}\rangle-\Gamma_{\beta} \vec{y}-\langle y, \vec{\varphi}\rangle, \\
& 0=\vec{e}_{n-2}^{*} \Gamma_{\beta} \vec{x}-\left\langle\vec{\varphi} \vec{x}+x, a_{n-1} \varphi_{n-1}\right\rangle+\left\langle x, \varphi_{n-2}\right\rangle-\left\langle\vec{\varphi} \vec{y}+y, \varphi_{n-1}\right\rangle .
\end{aligned}
$$

These equalities were obtained from (4.5) by setting

$$
\begin{array}{lll}
\vec{u}=0 & \text { and } & u_{n-1}=0, \\
u_{n-1}=0 & \text { and } & u_{r}=0, \\
u_{r}=0 & \text { and } & \vec{u}=0,
\end{array}
$$

respectively. From (4.6) and

$$
L \vec{\varphi}=\vec{\varphi} \mathfrak{M}+\varphi \vec{e}_{1}^{*}
$$

we obtain $0=(L x-y)+\vec{\varphi}(\mathfrak{M} \vec{x}-\vec{y})+\varphi x_{1}$. If we write $x_{n-1}=-(\mathfrak{M} \vec{x}-\vec{y})_{n-2}$ and use that the elements $\varphi, \varphi_{1}, \ldots, \varphi_{n-3}$ are linearly independent modulo $\mathcal{H}_{n-4}$, we find that

$$
x_{1}=0, \quad \vec{y}=\mathfrak{M} \vec{x}+x_{n-1} \vec{e}_{n-2},
$$

and $L x-y=x_{n-1} \varphi_{n-2}$. This last equality can be written as

$$
\left(L+a_{n-1}\right) x=y+a_{n-1} x+x_{n-1} \varphi_{n-2},
$$

which implies that

$$
x=x_{r}+x_{n-1} \varphi_{n-1}
$$

with

and

$$
x_{r}=\frac{1}{L+a_{n-1}}\left(y+a_{n-1} x\right) \in \mathcal{H}_{n}
$$

$$
y=L x_{r}-a_{n-1} x_{n-1} \varphi_{n-1} .
$$

Hence $\{X, Y\} \in B_{\max }$. We substitute (4.10),(4.11), and (4.12) in (4.7) and use (4.9) and we obtain that

$$
\begin{aligned}
0= & \left(\mathfrak{M}^{*} \Gamma_{\beta}-\Gamma_{\beta} \mathfrak{M}\right) \vec{x}+\left\langle x_{r}, \varphi \mathfrak{M}-L \vec{\varphi}\right\rangle \\
\quad & \quad+x_{n-1}\left\{\left\langle\varphi_{n-1}, \vec{\varphi}\left(\mathfrak{M}+a_{n-1}\right)\right\rangle-\Gamma_{\beta} \vec{e}_{n-2}\right\} \\
= & \left(\mathfrak{M}^{*} \Gamma_{\beta}-\Gamma_{\beta} \mathfrak{M}\right) \vec{x}-\left\langle x_{r}, \varphi\right\rangle \vec{e}_{1}+x_{n-1}\left(-\beta_{1, n-2}+\left(a_{1}-a_{n-1}\right) \beta_{1, n-1}\right) \vec{e}_{1} .
\end{aligned}
$$

If we apply $P$ to both sides and use that $x_{1}=0$ we get

$$
P\left(\Gamma_{\beta} \mathfrak{M}-\mathfrak{M}^{*} \Gamma_{\beta}\right) P \vec{x}=0
$$

and if we take the inner product in $\mathbb{C}^{n-2}$ on both sides with $\vec{e}_{1}$ we see $F_{\beta}(X)=0$. Finally, in the same way, if substitute (4.10), (4.11), and (4.12) in the righthand side of (4.8) and use (4.9) and (4.3) with $k=n-2$, we get after some calculations and cancellations that it is equal to

$$
\left\langle\varphi_{n-1}, \vec{\varphi}\left(\mathfrak{M}+a_{n-1}\right)\right\rangle-\vec{e}_{n-2}{ }^{*} \Gamma_{\beta} \vec{x}=-\beta_{n-2} \vec{e}_{1}^{*} \vec{x}
$$

and this equals 0 as $x_{1}=0$. In other words, (4.6) and (4.7) imply (4.8). The argument can easily be traced backwards to complete the proof that $B_{\max }^{+}$is the 
restriction of $B_{\max }$ as stated in the theorem. That $\left(B_{\max }^{+}\right)^{+}=B_{\max }$ can be verified in a similar way and this is left to the reader.

Recall that so far only the elements below the anti-diagonal of $\Gamma_{\beta}$ have been specified, see (4.2). From now on we assume in addition that

$$
P\left(\Gamma_{\beta} \mathfrak{M}-\mathfrak{M}^{*} \Gamma_{\beta}\right) P=0 .
$$

Under this condition one of the three "boundary conditions" determining $B_{\max }^{+}$as a restriction of $B_{\max }$ is always fulfilled. Together with the symmetry of the matrix $\Gamma_{\beta}$ the matrix equality (4.13) is equivalent to the relations

$$
\beta_{j, k-1}=\beta_{j-1, k}+\left(a_{k}-a_{j}\right) \beta_{j, k}, \quad j, k=2,3, \ldots, n-2, j+k \leq n .
$$

This implies that the not yet specified elements of $\Gamma_{\beta}$ are completely determined by the elements of the first row, which we can choose arbitrarily. Since we want the matrix $\Gamma_{\beta}$ to be real, we choose the entries $\beta_{1,1}, \ldots, \beta_{1, n-1} \in \mathbb{R}$. Under these conditions on $\Gamma_{\beta}$ we define the minimal operator

$$
B_{\min }:=B_{\max }^{+}=\left\{\left\{U, B_{\max } U\right\} \mid U \in \operatorname{dom} B_{\max }, u_{1}=0, F_{\beta}(U)=0\right\} .
$$

By Theorem 4.1 it is a densely defined symmetric operator on $\mathcal{H}_{B}$. The element

$$
\Phi_{B}(z)=\left(\begin{array}{c}
\vec{b}(z) \\
\frac{1}{L-z} \frac{b_{n-2}(z)}{b_{n-2}(L)} \varphi
\end{array}\right) \in \operatorname{dom} B_{\max }, \quad z \in \rho(L),
$$

satisfies the equation

$$
\left(B_{\max }-z\right) \Phi_{B}(z)=0,
$$

and so it is a defect element for $B_{\min }$. As in the $A$-model,

$$
\Phi_{B}(z)=\mathbf{j}_{1} \varphi(z), \quad \varphi(z)=\frac{1}{L-z} \varphi \in \mathbf{H} .
$$

Berezin's approach in [6] can be applied to describe all self-adjoint extensions $B$ (self-adjoint in the sense $B^{+}=B$ ) of $B_{\min }$ as a one-parameter family. Although $\mathcal{H}_{B}$ is a pre-Pontryagin space, the parametrization formula is the same as Krein's resolvent formula. To prepare for it we define a self-adjoint extension $B_{0}$ of $B_{\text {min }}$, show that $\Phi_{B}(z)$ is a defect function for $B_{0}$ and construct a $Q$-function for $B_{\text {min }}$ and $B_{0}$.

The self-adjoint extension $B_{0}$ of $B_{\min }$ which we choose to play the key role in the resolvent formula is given by

$$
\operatorname{dom} B_{0}=\left\{U \in \operatorname{dom} B_{\max } \mid u_{1}=0\right\}
$$

and

$$
B_{0}\left(\begin{array}{c}
0 \\
u_{2} \\
\vdots \\
u_{n-2} \\
u_{r}+u_{n-1} \varphi_{n-1}
\end{array}\right)=\left(\begin{array}{c}
u_{2} \\
u_{3}-a_{2} u_{2} \\
\vdots \\
u_{n-1}-a_{n-2} u_{n-2} \\
L u_{r}-a_{n-1} u_{n-1} \varphi_{n-1}
\end{array}\right)
$$


Then $B_{\min }$ can be interpreted as the one-dimensional restriction of $B_{0}$ to the domain

Note that

$$
\left\{U \in \operatorname{dom} B_{0} \mid\left\langle\left(B_{0}+a_{1}\right) U, \Phi_{B}\left(-a_{1}\right)\right\rangle_{B}=0\right\}
$$

$$
\Phi_{B}\left(-a_{1}\right)=\left(\begin{array}{c}
\vec{e}_{1} \\
0
\end{array}\right)
$$

We have $\rho\left(B_{0}\right)=\rho(L)$ and, for $z$ in this set, $\Phi_{B}(z)$ in (4.14) can be represented as

$$
\Phi_{B}(z)=\Phi_{B}\left(-a_{1}\right)+\left(z+a_{1}\right) \frac{1}{B_{0}-z} \Phi_{B}\left(-a_{1}\right) .
$$

This implies the Hilbert identity

$$
\Phi_{B}(z)-\Phi_{B}(\zeta)=(z-\zeta) \frac{1}{B_{0}-z} \Phi_{B}(\zeta)
$$

which together with the property $\Phi_{B}(z) \in \operatorname{ker}\left(B_{\max }-z\right)$ yield that $\Phi_{B}(z)$ is a defect function and

$$
Q_{B}(z)=\left(z+a_{1}\right)\left\langle\Phi_{B}(z), \Phi_{B}\left(-a_{1}\right)\right\rangle_{B},
$$

is a $Q$-function for $B_{\min }$ and $B_{0}$. Note that $Q_{B}(z)$ is normalized by the condition $Q_{B}\left(-a_{1}\right)=0$. Substituting the coordinates of $\Phi_{B}(z)$ in (4.15) we obtain that

$$
Q_{B}(z)=b_{n-2}(z)\left\langle\frac{z+a_{n-1}}{L-z} \varphi, \frac{1}{b_{n-1}(L)} \varphi\right\rangle+p_{n-2}(z),
$$

where with $\beta_{j}$ as in (4.4) the polynomial $p_{n-2}(z)$ is given by

$$
p_{n-2}(z)=\sum_{j=1}^{n-2} \beta_{j} b_{j}(z) \text {. }
$$

Using $B_{0}, \Phi_{B}(z)$ and $Q_{B}(z)$ we can now formulate the Berezin-Krein theorem which describes all self-adjoint extensions of $B_{\min }$ as a one-parameter family. The proof is similar to that of Theorem 3.3 and is therefore omitted.

Theorem 4.2. (i) The relation

$$
\frac{1}{B_{\theta}-z}=\frac{1}{B_{0}-z}-\frac{1}{Q_{B}(z)+\cot \theta}\left\langle\cdot, \Phi_{B}\left(z^{*}\right)\right\rangle_{B} \Phi_{B}(z) .
$$

defines a one-to-one correspondence between all self-adjoint extensions $B_{\theta}$ of $B_{\min }$ in $\mathcal{H}_{B}$ and the numbers $\theta \in[0, \pi)$.

(ii) The self-adjoint operator $B_{\theta}$ is the restriction of $B_{\max }$ described by the formula

$$
B_{\theta}=\left\{\left\{U, B_{\max } U\right\} \mid U \in \operatorname{dom} B_{\max }, \sin \theta F_{\beta}(\mathbf{U})=-\cos \theta u_{1}\right\} .
$$

The analogs of the formulas following Theorem 3.3 are

$$
B_{\max }=B_{0} \dot{+} \operatorname{span}\left\{\Phi_{B}\left(-a_{1}\right),-a_{1} \Phi_{B}\left(-a_{1}\right)\right\}
$$


and

$$
\begin{gathered}
B_{\theta}=\left\{\left\{U+u_{1} \Phi_{B}\left(-a_{1}\right), B_{0} U-a_{1} u_{1} \Phi_{B}\left(-a_{1}\right)\right\} \mid U \in \operatorname{dom} B_{0},\right. \\
\left.u_{1} \in \mathbb{C},\left\langle\left(B_{0}+a_{1}\right) U, \Phi_{B}\left(-a_{1}\right)\right\rangle_{B}=-u_{1} \cot \theta\right\} .
\end{gathered}
$$

\subsection{Compressions of the resolvent of $B_{\theta}$}

The following formula for the skew-compressed resolvent is valid

$$
\left.\mathbf{j}_{1}^{-1} \frac{1}{B_{\theta}-z}\right|_{\mathcal{H}_{n-2}}=\frac{1}{L-z}-\frac{1}{Q_{B}(z)+\cot \theta}\left\langle\cdot, \frac{1}{L-z^{*}} \varphi\right\rangle \frac{1}{L-z} \varphi,
$$

which is an analog of formula (3.13).

The formula for the compression of the resolvent $\frac{1}{B_{\theta}-z}$ to the subspace $\mathcal{H}_{n-2} \subset \mathcal{H}_{B}$ reads as follows:

$$
\begin{aligned}
P_{\mathcal{H}_{n-2}} & \left.\frac{1}{B_{\theta}-z}\right|_{\mathcal{H}_{n-2}}=\frac{1}{L-z} \\
& -\frac{1}{\left\langle\frac{z+a_{n-1}}{(L-z) b_{n-1}(L)} \varphi, \varphi\right\rangle+\frac{p_{n-2}(z)+\cot \theta}{b_{n-2}(z)}}\left\langle\cdot, \frac{1}{L-z^{*}} \varphi\right\rangle \frac{1}{L-z} \varphi_{n-2} .
\end{aligned}
$$

It implies that the spectral problem for the operator $B_{\theta}$ is equivalent to the following explicit eigenvalue depending "boundary value" problem:

$$
\begin{aligned}
(L-z) u & =c \varphi_{n-1}, \quad u \in \mathcal{H}_{n}, c \in \mathbb{C}, \\
b_{n-1}(z)\langle u, \varphi\rangle & =-c\left(p_{n-2}(z)+\cot \theta\right) .
\end{aligned}
$$

4.3. Pontryagin space completion and the self-adjoint realizations $\widehat{B}_{\theta}$

We set $\kappa=[(n-1) / 2]$. The inner product $(4.1)$ on $\mathcal{H}_{B}$ can be written also in the form

$$
\begin{aligned}
\left\langle\left(\begin{array}{l}
\vec{u} \\
u
\end{array}\right),\left(\begin{array}{l}
\vec{v} \\
v
\end{array}\right)\right\rangle_{B}= & \left\langle\left(\begin{array}{c}
\vec{u}^{\prime} \\
P_{\kappa} \vec{u}
\end{array}\right),\left(\begin{array}{cc}
0 & I_{\mathbf{C}^{\kappa}} \\
I_{\mathbf{C}^{\kappa}} & \left\{\beta_{j, k}\right\}_{j, k=1}^{\kappa}
\end{array}\right)\left(\begin{array}{c}
\vec{v}^{\prime} \\
P_{\kappa} \vec{v}
\end{array}\right)\right\rangle_{\mathbb{C}^{\kappa} \oplus \mathbb{C}^{\kappa}} \\
& +\left\langle\left(u+\sum_{j=\kappa+1}^{n-2} u_{j} \varphi_{j}\right),\left(v+\sum_{j=\kappa+1}^{n-2} v_{j} \varphi_{j}\right)\right\rangle,
\end{aligned}
$$

where

$$
\begin{gathered}
P_{\kappa} \vec{u}=\left(\begin{array}{c}
u_{1} \\
u_{2} \\
\vdots \\
u_{\kappa}
\end{array}\right), \\
\vec{u}^{\prime}=\sum_{j=1}^{\kappa}\left(\left\langle u, \varphi_{j}\right\rangle+\sum_{k=\kappa+1}^{n-2} u_{k} \beta_{j, k}\right) \vec{e}_{j}, \\
\vec{v}^{\prime}=\sum_{j=1}^{\kappa}\left(\left\langle v, \varphi_{j}\right\rangle+\sum_{k=\kappa+1}^{n-2} v_{k} \beta_{j, k}\right) \vec{e}_{j},
\end{gathered}
$$


and $\vec{e}_{1}, \ldots, \vec{e}_{\kappa}$ now stand for the standard basis in $\mathbb{C}^{\kappa}$. As the Gram matrix in the first inner product in (4.17) has $\kappa$ negative eigenvalues, the inner product on $\mathcal{H}_{B}$ has $\kappa$ negative squares. The Pontryagin space completion of $\mathcal{H}_{B}$ with respect to this inner product is the Pontryagin space $\widehat{\mathcal{H}}_{B}=\left(\mathbb{C}^{\kappa} \dot{+} \mathbb{C}^{\kappa}\right) \oplus \mathcal{H}$ with inner product, still be denoted by $\langle\cdot, \cdot\rangle_{B}$, defined by Gram operator

$$
G_{B}=\left(\begin{array}{cc}
0 & I_{\mathbf{C}^{\kappa}} \\
I_{\mathbf{C}^{\kappa}} & \left\{\beta_{j, k}\right\}_{j, k=1}^{\kappa}
\end{array}\right) \oplus I_{\mathcal{H}} .
$$

The natural isometric embedding $\mathbf{j}_{2}$ from $\mathcal{H}_{B}$ into its completion $\widehat{\mathcal{H}}_{B}$ is described by the formula

$$
\mathbf{j}_{2}\left(\begin{array}{c}
u_{1} \\
u_{2} \\
\vdots \\
u_{n-2} \\
u
\end{array}\right)=\left(\begin{array}{c}
\left(\left\langle u, \varphi_{j}\right\rangle+\sum_{k=\kappa+1}^{n-2} u_{k} \beta_{j, k}\right)_{j=1}^{\kappa} \\
\left(u_{j}\right)_{j=1}^{\kappa} \\
u+\sum_{j=\kappa+1}^{n-2} u_{j} \varphi_{j}
\end{array}\right) .
$$

The results proved for the operators in the pre-Pontryagin space $\mathcal{H}_{B}$ can be carried over to the closures of these operators in the Pontryagin space $\widehat{\mathcal{H}}_{B}$; see $[31,17,13]$.

Theorem 4.3. (i) The closure $\widehat{B}_{\min }$ of $\mathbf{j}_{2} B_{\min } \mathbf{j}_{2}^{-1}$ in $\widehat{\mathcal{H}}_{B}$ is a non-densely defined symmetric operator with defect indices $(1,1)$. Its adjoint $\widehat{B}_{\max }$ is the closure of $\mathbf{j}_{2} B_{\max } \mathbf{j}_{2}^{-1}$ in $\widehat{\mathcal{H}}_{B}$.

(ii) The closure $\widehat{B}_{0}$ of $\mathbf{j}_{2} B_{0} \mathbf{j}_{2}^{-1}$ in $\widehat{\mathcal{H}}_{B}$ is a self-adjoint relation with multi-valued part $\widehat{B}_{0}(0)=\operatorname{span}\left\{0 \oplus \vec{e}_{1} \oplus 0\right\}$. It is an extension of $\widehat{B}_{\min }$.

(iii) The function $\mathbf{j}_{2} \Phi_{B}(z)$ is the defect function and the function

$$
Q_{B}(z)=\left(z+a_{1}\right)\left\langle\Phi_{B}(z), \Phi_{B}\left(-a_{1}\right)\right\rangle_{B}
$$

is the $Q$-function for the operators $\widehat{B}_{\min }$ and $\widehat{B}_{0}$.

(iv) The formula

$$
\frac{1}{\widehat{B}_{\theta}-z}=\frac{1}{\widehat{B}_{0}-z}-\frac{1}{Q_{B}(z)+\cot \theta}\left\langle\cdot, \mathbf{j}_{2} \Phi_{B}\left(z^{*}\right)\right\rangle_{B} \mathbf{j}_{2} \Phi_{B}(z) .
$$

gives a one-to-one correspondence between all selfadjoint extensions $\widehat{B}_{\theta}$ of $\widehat{B}_{\min }$ in $\widehat{\mathcal{H}}_{B}$ and $\theta \in[0, \pi)$. Each $\widehat{B}_{\theta}$ is the closure of $\mathbf{j}_{2} B_{\theta} \mathbf{j}_{2}^{-1}$ in $\widehat{\mathcal{H}}_{B}$.

\subsection{On properties of the function $Q_{B}(z)$}

The function $Q_{B}(z)$ is a generalized Nevanlinna function which belongs to the class $N_{\kappa}, \kappa=[(n-1) / 2]$. More precisely, $Q_{B}(z)$ belongs to the subclass $N_{\kappa}^{\infty} \subset N_{\kappa}$ considered in [13]: $N_{\kappa}^{\infty}$ consists of the functions $Q(z) \in N_{\kappa}$ which are holomorphic on $\mathbf{C} \backslash \mathbf{R}$ and admit the representation

$$
Q(z)=(z+1)^{2 \kappa} Q_{0}(z)+p_{2 \kappa-1}(z),
$$


where $Q_{0}(z) \in N_{0}$,

$$
\lim _{y \rightarrow \infty} \frac{\operatorname{Im} Q_{0}(i y)}{y}=0, \quad \lim _{y \rightarrow \infty} y \operatorname{Im} Q_{0}(i y)=\infty,
$$

and $p_{2 \kappa-1}(z)$ is a real polynomial of degree at most $2 \kappa-1$.

In this case, since $L$ is a nonnegative operator, one can say more, see [14]: The function $Q_{0}(z)$ is holomorphic on $\mathbf{C} \backslash \mathbf{R}^{+}$and its asymptotic behavior at $-\infty$ along the negative real axis is given by

$$
\lim _{x \rightarrow-\infty} Q_{0}(x)=\left\{\begin{array}{cl}
0, & \text { if } n \text { is odd, } \\
-\infty, & \text { if } n \text { is even. }
\end{array}\right.
$$

Writing $Q_{B}(z)+\cot \theta$ in the form (4.18):

$$
Q_{B}(z)+\cot \theta=(z+1)^{2 \kappa} Q_{0}(z)+p_{2 \kappa-1}(z),
$$

one can show that

$$
Q_{0}(z)=\left\{\begin{array}{cl}
\left\langle\frac{1}{(L-z)(L+1)^{\kappa}} \varphi, \frac{1}{(L+1)^{\kappa}} \varphi\right\rangle, & \text { if } n \text { is odd, } \\
\left\langle\frac{z+1}{(L-z)(L+1)^{\kappa}} \varphi, \frac{1}{(L+1)^{\kappa+1}} \varphi\right\rangle+g_{2 \kappa+1}, & \text { if } n \text { is even, }
\end{array}\right.
$$

is independent of $\theta$ and

$$
p_{2 \kappa-1}(z)=\sum_{j=0}^{2 \kappa-1} g_{j+1}(z+1)^{j} .
$$

Here

$$
g_{1}=Q_{B}(-1)+\cot \theta, \quad g_{k}=\frac{1}{(k-1) !} Q_{B}^{(k-1)}(-1), \quad k=2,3, \ldots, 2 \kappa+1,
$$

and by calculating the derivatives these numbers can be expressed in terms of the normalization points $a_{j}$ and the parameters $\beta_{1, j}, j=1,2, \ldots, n-2$.

\subsection{Compression of the resolvent of $\widehat{B}_{\theta}$}

The function $Q_{B}(z)$ admits not only the representation (4.18) but also the representation (see [13, Section 6], where the relations between various representations are described):

$$
Q_{B}(z)=b_{\kappa}(z)^{2} \widehat{Q}_{0}(z)+\widehat{p}_{2 \kappa-1}(z),
$$

with Nevanlinna function

$$
\widehat{Q}_{0}(z)=\left\{\begin{array}{cc}
\left\langle\frac{1}{(L-z) b_{\kappa}(L)} \varphi, \frac{1}{b_{\kappa}(L)} \varphi\right\rangle, & n=2 \kappa+1, \\
\left\langle\frac{z+a_{\kappa+1}}{(L-z) b_{\kappa}(L)} \varphi, \frac{1}{b_{\kappa+1}(L)} \varphi\right\rangle+\beta_{\kappa+1, \kappa}, & n=2 \kappa+2,
\end{array}\right.
$$


and polynomial

$$
\widehat{p}_{2 \kappa-1}(z)=\sum_{j=1}^{\kappa} \beta_{1, j}\left(z+a_{1}\right) b_{j-1}(z)+\sum_{j=2}^{\kappa} \beta_{\kappa, j} b_{j-1}(z) b_{\kappa}(z) .
$$

Then by Krein's formula in Theorem 4.2 (i), the formula for the compression of the resolvent of $\widehat{B}_{\theta}$ to $\mathcal{H}$ takes the form

$$
\left.P_{\mathcal{H}} \frac{1}{\widehat{B}_{\theta}-z}\right|_{\mathcal{H}}=\frac{1}{L-z}-\frac{1}{\widehat{Q}_{0}(z)+\tau_{\theta}(z)}\left\langle\cdot, \frac{1}{L-z^{*}} \widehat{\varphi}\right\rangle \frac{1}{L-z} \widehat{\varphi},
$$

where $\widehat{\varphi}:=\frac{1}{b_{\kappa}(L)} \varphi$, and

$$
\tau_{\theta}(z):=\frac{\widehat{p}_{2 \kappa-1}(z)+\cot \theta}{b_{\kappa}(z)^{2}} .
$$

This compressed resolvent is a generalized resolvent of the one-dimensional restriction of $L$ in $\mathcal{H}$ :

$$
\widehat{B}_{\min }:=\left\{\{u, L u\} \mid u \in \mathcal{H}_{2},\langle u, \widehat{\varphi}\rangle=0\right\} .
$$

The adjoint of this restriction is given by

$$
\widehat{B}_{\max }:=\widehat{B}_{\min }^{*}=\left\{\left\{u+c \frac{1}{L+a_{\kappa+1}} \widehat{\varphi}, L u-c \frac{a_{\kappa+1}}{L+a_{\kappa+1}} \widehat{\varphi}\right\} \mid u \in \mathcal{H}_{2}, c \in \mathbb{C}\right\} .
$$

It implies that the spectral problem for the operator $\widehat{B}_{\theta}$ is equivalent to the following explicit eigenvalue depending "boundary value" problem in $\mathcal{H}$ :

$$
\begin{aligned}
(L-z) u & =c \frac{1}{L+a_{\kappa+1}} \varphi, \quad u \in \mathcal{H}_{2}, c \in \mathbb{C}, \\
\left(z+a_{\kappa+1}\right)\langle u, \widehat{\varphi}\rangle & =-c\left(\widehat{Q}_{0}\left(-a_{\kappa+1}\right)+\tau_{\theta}(z)\right) .
\end{aligned}
$$

\section{Comparison of the models}

Evidently, there is a close relation between the $A$ - and $B$ - models. The aim of this section is to describe what the two have in common and to point out their differences. Among other things we discuss the dependence of the models on the parameters and compare aspects of the negative point spectra of the self-adjoint operators.

\subsection{Minimality of the models}

The operator representations of the functions $Q_{A}(z)$ and $Q_{B}(z)$ in the models are unique up to unitary equivalence if

$$
\mathcal{H}_{A}=\overline{\operatorname{span}}\left\{\Phi_{A}(z) \mid z \in \rho(L)\right\}
$$

and

$$
\widehat{\mathcal{H}}_{B}=\overline{\operatorname{span}}\left\{\mathbf{j}_{2} \Phi_{B}(z) \mid z \in \rho(L)\right\}
$$


respectively. These minimality conditions can be achieved simultaneously by requiring that the interaction is cyclic with respect to $L$, that is,

$$
\mathcal{H}=\overline{\operatorname{span}}\left\{\frac{1}{(L-z) b_{n-1}(L)} \varphi \mid z \in \rho(L)\right\} .
$$

This implies also that

$$
\mathbf{H}=\overline{\operatorname{span}}\left\{\frac{1}{L-z} \varphi \mid z \in \rho(L)\right\} .
$$

\subsection{The parameters of the models}

We first list the parameters used in the two models:

Model $A$ :

- the normalization points $a_{1}, a_{2}, \ldots, a_{n-1}$,

- the real numbers $\alpha_{1, n-2}, \alpha_{2, n-2}, \ldots, \alpha_{n-2, n-2}$ in the last column of $\Gamma_{\alpha}$,

- the self-adjoint extension parameter $\theta_{A} \in[0, \pi)$.

Model $B$ :

- the normalization points $a_{1}, a_{2}, \ldots, a_{n-1}$,

- the real numbers $\beta_{1,1}, \beta_{1,2}, \ldots, \beta_{1, n-2}$ in the first row of $\Gamma_{\beta}$,

- the self-adjoint extension parameter $\theta_{B} \in[0, \pi)$.

For a given set of normalization points the other parameters are independent and parameterize the models in an unique way. Therefore the two models will be compared in the case where the sets of normalization points are the same.

Since the functions in the denominators in Krein's formulas determine the corresponding operators uniquely up to a unitary transformation, it is enough to compare these two functions

$$
Q_{A}(z)+\cot \theta_{A} \text { and } Q_{B}(z)+\cot \theta_{B} .
$$

Indeed, for $\theta \neq 0$, the functions

$$
-\frac{1}{Q_{A}(z)+\cot \theta}, \quad-\frac{1}{Q_{B}(z)+\cot \theta}
$$

are the $Q$-functions of $A_{\theta}$ and $A_{\min }$ and $B_{\theta}$ and $B_{\min }$ respectively. It is more convenient to compare the following two functions instead:

$$
\begin{aligned}
& b_{n-2}(z)\left(Q_{A}(z)+\cot \theta_{A}\right) \\
& \quad=b_{n-2}(z)\left\langle\frac{z+a_{n-1}}{L-z} \varphi, \frac{1}{b_{n-1}(L)} \varphi\right\rangle-\sum_{k=1}^{n-2} \alpha_{k, n-2} b_{k-1}(z)-\alpha b_{n-2}(z),
\end{aligned}
$$

where

$$
\alpha=\cot \theta_{A}+\sum_{k=1}^{n-2} \alpha_{k, n-2} \frac{b_{k-1}\left(-a_{n-1}\right)}{b_{n-2}\left(-a_{n-1}\right)},
$$


and

$$
\begin{aligned}
& Q_{B}(z)+\cot \theta_{B} \\
& \quad=b_{n-2}(z)\left\langle\frac{z+a_{n-1}}{L-z} \varphi, \frac{1}{b_{n-1}(L)} \varphi\right\rangle+\sum_{k=1}^{n-2} \beta_{j} b_{j}(z)+\cot \theta_{B} .
\end{aligned}
$$

These two functions coincide if and only if the parameters are related as follows

$$
\left(\begin{array}{c}
\cot \theta_{B} \\
\beta_{1} \\
\vdots \\
\beta_{n-3} \\
\beta_{n-2}
\end{array}\right)=\operatorname{diag}(-1,-1, \ldots,-1,1)\left(\begin{array}{c}
\alpha_{1, n-2} \\
\alpha_{2, n-2} \\
\vdots \\
\alpha_{n-2, n-2} \\
\cot \theta_{A}
\end{array}\right)
$$

Recall that the numbers $\beta_{j}, j=1,2, \ldots, n-2$ given by (4.4). This formula shows that there is a one-to-one correspondence between these numbers and the $n-2$ entries of the first row of the matrix $\Gamma_{\beta}$ :

$$
\left(\begin{array}{c}
\beta_{1} \\
\beta_{2} \\
\vdots \\
\beta_{n-3} \\
\beta_{n-2}
\end{array}\right)=\left(\begin{array}{ccccc}
1 & a_{1}-a_{2} & \ldots & 0 & 0 \\
0 & 1 & \ddots & & 0 \\
\vdots & \ddots & \ddots & \ddots & \vdots \\
0 & & \ddots & 1 & a_{1}-a_{n-3} \\
0 & 0 & \ldots & 0 & 1
\end{array}\right)\left(\begin{array}{c}
\beta_{1,1} \\
\beta_{1,2} \\
\vdots \\
\beta_{1, n-3} \\
\beta_{1, n-2}
\end{array}\right)+\beta_{1, n-1}\left(\begin{array}{c}
0 \\
0 \\
\vdots \\
0 \\
a_{1}-a_{n-1}
\end{array}\right) .
$$

In the last summand on the righthand side

$$
\beta_{1, n-1}=\left\langle\frac{1}{b_{n-1}(L)} \varphi, \frac{1}{L+a_{1}} \varphi\right\rangle
$$

and, since the matrix in (5.4) is invertible, the formulas (5.3) and (5.4) describe a one-to-one correspondence between the parameters in the models $A$ and $B$, except in the cases where $\theta_{A}=0$ or $\theta_{B}=0$. In these exceptional cases, the self-adjoint operator $A_{0}$ has no counterpart in the $B$-model and the self-adjoint relation $\widehat{B}_{0}$ has no counterpart in the $A$-model.

Theorem 5.1. Assume that the normalization points in the $A$ - and B-models are the same and that the parameters $\alpha_{j, n-2}$ and $\theta_{A} \neq 0$ and the parameters $\beta_{1, j}$ 's and $\theta_{B} \neq 0$ of the two models are related by the equations (5.3) and (5.4). Then the identification map $T=\mathbf{j}_{1} \mathbf{i}: \mathcal{H}_{A} \rightarrow \mathcal{H}_{B}$ intertwines the self-adjoint operators $A_{\theta_{A}}$ in Theorem 3.3 and $B_{\theta_{B}}$ in Theorem 4.2:

$$
B_{\theta_{B}} T=T A_{\theta_{A}} \text {. }
$$

Remark 5.2. If we provide $\mathcal{H}_{B}$ with a new norm that makes $\mathbf{j}_{1}$ an isomorphism, then the operators $B_{\max }$ and its self-adjoint restrictions are closed, $T$ is a similarity operator, that is, bounded and boundedly invertible, and the intertwining relation (5.5) shows that the operators $A_{\theta_{A}}$ and $B_{\theta_{B}}$ are similar. 
Proof of Theorem 5.1. By definition,

$$
T A_{\max }=\mathbf{j}_{1} \mathbf{L}_{\max } \mathbf{i}=B_{\max } T .
$$

The relations (5.3) and (5.4) readily imply $T \operatorname{dom} A_{\theta_{A}} \subset \operatorname{dom} B_{\max }$ and that the restriction (3.11) describing $A_{\theta_{A}}$ which can be written as

$$
\left\langle u_{r}, \varphi\right\rangle+\cot \theta_{A} u_{n-1}-\sum_{j=1}^{n-2} \alpha_{j, n-2} u_{j}=0
$$

is equivalent to the restriction

$$
\left\langle u_{r}, \varphi\right\rangle+\sum_{k=1}^{n-2} \beta_{j} u_{j+1}+\cot \theta_{B} u_{1}=0
$$

in the formula for (4.16) for $B_{\theta_{B}}$. Hence $T \operatorname{dom} A_{\theta_{A}}=\operatorname{dom} B_{\theta_{B}}$, and now the intertwining formula (5.5) easily follows.

\subsection{The spectra of the realizations}

Assume the conditions of Theorem 5.1 and assume that $\varphi$ is a cyclic generalized element for $L$. Then Theorem 5.1 implies that

$$
\rho\left(A_{\theta_{A}}\right)=\rho\left(B_{\theta_{B}}\right), \quad \sigma_{c}\left(A_{\theta_{A}}\right)=\sigma_{c}\left(B_{\theta_{B}}\right), \quad \sigma_{p}\left(A_{\theta_{A}}\right)=\sigma_{p}\left(B_{\theta_{B}}\right) .
$$

As the resolvent $\left(\widehat{B}_{\theta_{B}}-z\right)^{-1}, z \in \rho\left(B_{\theta_{B}}\right)$, coincides with the closure in $\widehat{\mathcal{H}}_{B}$ of the resolvent $\left(B_{\theta_{B}}-z\right)^{-1}$ it follows that $\rho\left(\widehat{B}_{\theta_{B}}\right)=\rho\left(B_{\theta_{B}}\right)$, and, therefore the spectra of $\widehat{B}_{\theta_{B}}$ and $B_{\theta_{B}}$ coincide. Also for the essential spectrum of $\widehat{B}_{\theta_{B}}$ we have the equalities

$$
\sigma_{\mathrm{ess}}\left(\widehat{B}_{\theta_{B}}\right)=\sigma_{\mathrm{ess}}\left(\widehat{B}_{0}\right)=\sigma_{\mathrm{ess}}(L)=\sigma_{\mathrm{ess}}\left(A_{0}\right)=\sigma_{\mathrm{ess}}\left(A_{\theta_{A}}\right),
$$

and hence $\sigma_{\text {ess }}\left(\widehat{B}_{\theta_{B}}\right) \subset \overline{\mathbb{R}^{+}}$. These equalities follow from the fact that the resolvents of $A_{\theta_{A}}$ and $\widehat{B}_{\theta_{B}}$ are rank one perturbations of the resolvents of $A_{0}$ and $\widehat{B}_{0}$, respectively. Hence the parts in $\mathbb{C} \backslash \overline{\mathbb{R}^{+}}$of $\sigma\left(\widehat{B}_{\theta_{B}}\right)$ and $\sigma\left(A_{\theta_{A}}\right)$ are the same. Recall that for $\theta_{A}, \theta_{B} \neq 0$,

$$
-\left(Q_{A}(z)+\cot \theta_{A}\right)^{-1}=-b_{n-2}(z)\left(Q_{B}(z)+\cot \theta_{B}\right)^{-1}
$$

where the functions

$$
-\left(Q_{A}(z)+\cot \theta_{A}\right)^{-1}, \quad-\left(Q_{B}(z)+\cot \theta_{B}\right)^{-1}
$$

are the $Q$-functions of $A_{\theta_{A}}$ and $A_{\min }$ and $\widehat{B}_{\theta_{B}}$ and $B_{\min }$, respectively, which determine the extensions $A_{\theta_{A}}$ and $\widehat{B}_{\theta_{B}}$ up to unitary equivalence. We apply to both sides of (5.6) Langer's criterion [23, Theorem 1], which characterizes the eigenvalues of non-positive and positive type of $A_{\theta_{A}}$ and $\widehat{B}_{\theta_{B}}$ in terms of certain non-tangential limits for the generalized Nevanlinna functions in (5.7); the multiplier $b_{n-2}(z)$ on the righthand side of (5.6) plays no role in these limits, because $b_{n-2}(x)>0$ for $x \geq 0$. We obtain that the eigenvalues of non-positive (positive) type of $\widehat{B}_{\theta_{B}}$ in 
$\overline{\mathbb{R}^{+}}$are the eigenvalues of non-positive type (positive type, respectively) of $A_{\theta_{A}}$. It follows that the continuous and point spectra of $A_{\theta_{A}}$ and $\widehat{B}_{\theta_{B}}$ coincide:

$$
\sigma_{c}\left(A_{\theta_{A}}\right)=\sigma_{c}\left(\widehat{B}_{\theta_{B}}\right), \quad \sigma_{p}\left(A_{\theta_{A}}\right)=\sigma_{p}\left(\widehat{B}_{\theta_{B}}\right) .
$$

We consider the case where $\mathcal{H}_{A}$ is a Hilbert space in more detail.

Theorem 5.3. Assume that the normalization points are mutually different and ordered such that $0<a_{1}<a_{2}<\ldots<a_{n-2}, \Gamma_{\alpha}>0$, and that the conditions of Theorem 5.1 hold. Then $\sigma_{p}\left(A_{\theta_{A}}\right)=\sigma_{p}\left(\widehat{B}_{\theta_{B}}\right)$ contains at least $n-2$ simple eigenvalues $z_{j}$ with $-a_{j+1}<z_{j}<-a_{j}, j=1,2, \ldots, n-3$ and $z_{n-2}<a_{n-2}$. The ones with an even index are of positive type and those with an odd index are of negative type for the operator $\widehat{B}_{\theta}$.

Proof. The function $Q_{A}(z)$ is a Nevanlinna function of the form

$$
Q_{A}(z)=\left\langle\frac{z+a_{n-1}}{L-z} \varphi, \frac{1}{b_{n-1}(L)} \varphi\right\rangle-\sum_{j=1}^{n-2} \frac{\sigma_{j}}{z+a_{j}},
$$

which has only real zeros. Introduce the disjoint intervals

$$
I_{n-2}=\left(-\infty,-a_{n-2}\right), I_{n-3}=\left(-a_{n-2},-a_{n-3}\right), \ldots, I_{1}=\left(-a_{2},-a_{1}\right) .
$$

As each point $-a_{j}, j=1,2, \ldots, n-2$ is a pole of the function $Q_{A}(z)+\cot \theta_{A}$ and $\lim _{x \rightarrow-\infty} Q_{A}(x)=-\infty$, this function has exactly one zero, say $z_{j}$, in each interval $I_{j}, j=1,2, \ldots, n-2$. Hence $\sigma_{p}\left(A_{\theta_{A}}\right)=\sigma_{p}\left(\widehat{B}_{\theta_{B}}\right)$ contains at least $n-2$ negative simple eigenvalues, namely $z_{1}, z_{2}, \ldots, z_{n-2}$. We prove that for the generalized Nevanlinna function $Q_{B}(z)+\cot \theta_{B}$ the zeros $z_{2 k}$ with even index are zeros of positive type and the exactly $\kappa$ zeros $z_{2 k-1}$ with odd index are zeros of negative type, $k=1,2, \ldots,[(n-2) / 2]$. Indeed, since $z_{1}, z_{2}, \ldots, z_{n-2}$ are simple eigenvalues of $\widehat{B}_{\theta_{B}}$ and by [13, Theorem 3.3], the vectors $\mathbf{j}_{2} \Phi_{B}\left(z_{1}\right), \mathbf{j}_{2} \Phi_{B}\left(z_{2}\right), \ldots, \mathbf{j}_{2} \Phi_{B}\left(z_{n-2}\right)$ are the corresponding eigenvectors and

$$
\left\langle\boldsymbol{\Phi}_{B}\left(z_{j}\right), \boldsymbol{\Phi}_{B}\left(z_{j}\right)\right\rangle_{B}=Q_{B}^{\prime}\left(z_{j}\right),
$$

where $Q_{B}^{\prime}(z)$ means derivative of $Q_{B}(z)$ in $z$. According to (5.1) and (5.2),

$$
Q_{B}^{\prime}\left(z_{j}\right)=\prod_{k=1}^{j}\left(a_{k}-z_{j}\right) \prod_{k=j+1}^{n-2}\left(a_{k}-z_{j}\right) Q_{A}^{\prime}\left(z_{j}\right) .
$$

As $Q_{A}(z) \in N_{0}$ we have that $Q_{A}^{\prime}\left(z_{j}\right)>0$ for $j=1,2, \ldots, n-2$. Since $z_{j}<-a_{j}$, the second product is positive and the first product is positive, if $j$ is even, and it is negative, if $j$ is odd.

Further, according to a theorem of L.S. Pontryagin each self-adjoint operator $B$ in Pontryagin space $\Pi_{\kappa}$ with $\kappa$ negative squares has a $\kappa$-dimensional non-positive invariant subspace $\mathcal{M}(B)$ such that the spectrum $\sigma\left(\left.B\right|_{\mathcal{M}(B)}\right)$ of the restriction of $B$ to $\mathcal{M}(B)$ is in the closed upper half plane. In the case of the Theorem 5.3,

$$
\mathcal{M}=\operatorname{span}\left\{\mathbf{j}_{2} \Phi_{B}\left(z_{1}\right), \mathbf{j}_{2} \Phi_{B}\left(z_{3}\right), \ldots, \mathbf{j}_{2} \Phi_{B}\left(z_{2 \kappa-1}\right)\right\}
$$


and the space $\widehat{\mathcal{H}}_{B}$ admits the orthogonal decomposition

$$
\widehat{\mathcal{H}}_{B}=\mathcal{H}_{+} \oplus \mathcal{M}
$$

where $\mathcal{H}_{+}$is a $\widehat{B}_{\theta_{B}}$-invariant Hilbert subspace of $\widehat{\mathcal{H}}_{B}$. The restriction $B_{+}:=$ $\widehat{B}_{\theta_{B}} \mid \mathcal{H}_{+}$is a self-adjoint operator in $\mathcal{H}_{+}$and describes the Hilbert space part of the $\mathrm{B}$-model. This operator is not similar to $A_{\theta_{A}}$, since

$$
\sigma\left(B_{+}\right)=\sigma\left(A_{\theta_{A}}\right) \backslash\left\{z_{1}, z_{3}, \ldots, z_{2 \kappa-1}\right\} .
$$

The paper concerns the realization problem for highly singular perturbations and we describe two different models: A and B. In the situation of Theorem 5.3 the Amodel is a Hilbert space realization and the B-model is a Pontryagin realization. The negative type spectrum of $\widehat{B}_{\theta_{B}}$ in the B-model consists of simple isolated eigenvalues and hence $\widehat{B}_{\theta_{B}}$ is similar to a Hilbert space operator. In fact, the decomposition (5.8) implies that the operator $\widehat{B}_{\theta_{B}}^{\prime}:=\left.\left.\widehat{B}_{\theta_{B}}\right|_{\mathcal{H}_{+}} \oplus \widehat{B}_{\theta_{B}}\right|_{\mathcal{M}}$ is selfadjoint in the space $\widehat{\mathcal{H}}_{B}$ equipped with the positive scalar product

$$
\langle\cdot, \cdot\rangle^{\prime}=\langle\cdot, \cdot\rangle_{\mathcal{H}_{+}}-\langle\cdot, \cdot\rangle_{\mathcal{M}},
$$

which makes it a Hilbert space. The operator $\widehat{B}_{\theta_{B}}^{\prime}$ however, is not a solution of the realization problem and, from the point of view of scattering theory, its restriction to $\mathcal{H}_{+}$, that is, the operator $B_{+}$considered above, is the more appropriate Hilbert space operator.

\section{Examples}

Here we illustrate the main points in the correspondence between the $A$-model and the $B$-model in the simplest cases when $\varphi \in \mathcal{H}_{-n} \backslash \mathcal{H}_{-n+1}$ with $n=3$ and $n=4$. Concrete examples with $n=3$ are point-like perturbations of the Laplacian $L=-\Delta$ in $\mathcal{H}=L^{2}\left(\mathbb{R}^{4}\right)$ and $\mathcal{H}=L^{2}\left(\mathbb{R}^{5}\right)$ with the interaction $\varphi=\delta(x)$. Then the regularized $Q$-functions are of the form

$$
Q(z)=-\frac{1}{16 \pi^{2}} z \ln (-z)+c_{1} z+c_{0},
$$

and

$$
Q(z)=\frac{1}{24 \pi^{2}}(-z)^{3 / 2}+c_{1} z+c_{0},
$$

respectively, with real parameters $c_{0}, c_{1}$.

Examples with $n=4$ are point-like perturbations of $L=-\Delta$ in $\mathcal{H}=L^{2}\left(\mathbb{R}^{6}\right)$ and $\mathcal{H}=L^{2}\left(\mathbb{R}^{7}\right)$ also with the interaction $\varphi=\delta(x)$. Now the regularized $Q$-functions are

and

$$
Q(z)=-\frac{1}{128 \pi^{3}} z^{2} \ln (-z)+c_{2} z^{2}+c_{1} z+c_{0}
$$

$$
Q(z)=-\frac{1}{240 \pi^{3}}(-z)^{5 / 2}+c_{2} z^{2}+c_{1} z+c_{0},
$$


respectively, where $c_{0}, c_{1}, c_{2}$ are real parameters. In all four cases the functions $Q(z)$ are generalized Nevanlinna functions from the class $N_{1}^{\infty}$, that is, with 1 negative square and with the only one pole of non-positive type at $z=\infty$.

\subsection{Derivation of the formulas}

For $d=1,2, \ldots$, let $L$ be the self-adjoint realization of the Laplacian $-\Delta$ in $\mathcal{H}=$ $L^{2}\left(\mathbb{R}^{d}\right)$ and let $\varphi=\delta(x)$. Observe that $\varphi \in \mathcal{H}_{-n} \backslash \mathcal{H}_{1-n}$, where $n=2+[(d-2) / 2]$. This can be checked by using the spectral representation of $L$ in $L^{2}\left(\mathbb{R}^{+}\right) \otimes L^{2}\left(S_{d-1}\right)$ $\left(S_{d-1}\right.$ denotes the unit sphere in $\left.\mathbb{R}^{d}\right)$, where $L$ is realized as the operator of multiplication by the independent variable, say $\lambda$, and $\varphi=\delta(x)$ is represented by the function

$$
\widetilde{\varphi}(\lambda)=\left(\frac{1}{2^{d} \pi^{d / 2} \Gamma(d / 2)}\right)^{1 / 2} \lambda^{(d-2) / 4} .
$$

Here $\Gamma(\lambda)$ is the Euler gamma-function. Applying the regularization procedure from Subsection 1.4 with equal normalization points $a_{1}=a_{2}=\ldots=a_{n-1}=a>0$ to the formal expression $\left\langle\frac{1}{L-z} \varphi, \varphi\right\rangle$, we obtain the regularization

$$
Q(z)=(z+a)^{n-1}\left\langle\frac{1}{L-z} \varphi, \frac{1}{(L+a)^{n-1}} \varphi\right\rangle+\sum_{j=0}^{n-2} p_{j}(z+a)^{j},
$$

where the $p_{j}$ 's are real numbers. By the spectral representation of $L$, the first summand can be written as

$$
(z+a)^{n-1}\left\langle\frac{1}{L-z} \varphi, \frac{1}{(L+a)^{n-1}} \varphi\right\rangle=\frac{(z+a)^{n-1}}{2^{d} \pi^{d / 2} \Gamma\left(\frac{d}{2}\right)} \int_{0}^{\infty} \frac{\lambda^{(d-2) / 2} d \lambda}{(\lambda-z)(\lambda+a)^{n-1}} .
$$

For the integral, using a calculation as in the proof of [14, Proposition 2.1], we find

$$
(z+a)^{n-1} \int_{0}^{\infty} \frac{\lambda^{(d-2) / 2} d \lambda}{(\lambda-z)(\lambda+a)^{n-1}}=F(z)-\sum_{j=0}^{n-1} \frac{(z+a)^{j}}{j !} F^{(j)}(-a),
$$

where

$$
F(z)=\left\{\begin{aligned}
-\pi \frac{(-z)^{(d-2) / 2}}{\sin (\pi(d-2) / 2)}, & \text { if } d \text { is odd }, \\
-z^{(d-2) / 2} \ln (-z), & \text { if } d \text { is even } .
\end{aligned}\right.
$$

Inserting these results in the expression of $Q(z)$ we see that

$$
Q(z)=\left\{\begin{array}{cc}
-\frac{1}{2^{d} \pi^{(d-2) / 2} \Gamma(d / 2)} \frac{(-z)^{(d-2) / 2}}{\sin (\pi(d-2) / 2)}+p_{[(d-2) / 2]}(z), & \text { if } d \text { is odd, } \\
-\frac{1}{2^{d} \pi^{d / 2} \Gamma(d / 2)} z^{(d-2) / 2} \ln (-z)+p_{(d-2) / 2}(z), & \text { if } d \text { is even, }
\end{array}\right.
$$

where $p_{\ell}(z)$ stands for a polynomial of degree at most $\ell$ with real coefficients. The formulas for $Q(z)$ mentioned in the beginning of this section correspond to the cases $d=4,5,6$, and 7 , respectively. 
6.2. The case $n=3$

In this case $\varphi \in \mathcal{H}_{-3} \backslash \mathcal{H}_{-2}$ and there are two normalization points $a_{1}, a_{2}>0$. We set

$$
b_{1}(z)=z+a_{1}, b_{2}(z)=\left(z+a_{1}\right)\left(z+a_{2}\right), \quad \varphi_{j}=\frac{1}{b_{j}(L)} \varphi, j=1,2 .
$$

The $A$-model. The function $Q_{A}(z)+\cot \theta_{A}$ has the form

$$
Q_{A}(z)+\cot \theta_{A}=\left\langle\frac{z+a_{2}}{L-z} \varphi_{2}, \varphi\right\rangle-\frac{\alpha}{z+a_{1}}+\cot \theta_{A},
$$

where $\alpha \in \mathbb{R}$ and $\theta_{A} \in[0, \pi)$. The space of the self-adjoint realization of the singular perturbation is

$$
\mathcal{H}_{A}=\mathbb{C} \oplus \mathcal{H}_{1}
$$

with Gram operator $G_{A}=\alpha \oplus I_{\mathcal{H}_{1}} . G_{A}$ is non-degenerate only if $\alpha \neq 0, \mathcal{H}_{A}$ is a Hilbert space if $\alpha>0$, and it is a Pontryagin space with negative index 1 if $\alpha<0$. The self-adjoint realization in the $A$-model is the self-adjoint operator in the representation of the function $-\left(Q_{A}(z)+\cot \theta_{A}\right)^{-1}$ and this is the operator $A_{\theta_{A}}$ in $\mathcal{H}_{A}$ which is the restriction of

$$
A_{\max }\left(\begin{array}{c}
u_{1} \\
u_{r}+u_{2} \varphi_{2}
\end{array}\right)=\left(\begin{array}{c}
u_{2}-a_{1} u_{1} \\
L u_{r}-a_{2} u_{2} \varphi_{2}
\end{array}\right), \quad u_{r} \in \mathcal{H}_{3}, u_{1}, u_{2} \in \mathbb{C},
$$

by the self-adjoint "boundary" condition

$$
\left\langle u_{r}, \varphi\right\rangle-\alpha u_{1}=-\cot \theta_{A} u_{2} .
$$

The $B$-model. The function $Q_{B}(z)+\cot \theta_{B}$ has the form

$$
Q_{B}(z)+\cot \theta_{B}=b_{2}(z)\left\langle\frac{1}{L-z} \varphi_{2}, \varphi\right\rangle+\beta_{1}\left(z+a_{1}\right)+\cot \theta_{B},
$$

where $\beta_{1} \in \mathbb{R}$ and $\theta_{B} \in[0, \pi)$. The space of the realization is

$$
\widehat{\mathcal{H}}_{B}=\mathbb{C} \oplus \mathbb{C} \oplus \mathcal{H}
$$

with Gram operator

$$
G_{B}=\left(\begin{array}{cc}
0 & 1 \\
1 & \beta_{11}
\end{array}\right) \oplus I_{\mathcal{H}}, \quad \beta_{11}=\beta_{1}+\left(a_{2}-a_{1}\right)\left\langle\varphi_{2}, \varphi_{1}\right\rangle .
$$

It is a Pontryagin space with negative index 1 and the completion of the prePontryagin space $\mathcal{H}_{B}=\mathbb{C} \dot{+} \mathcal{H}_{1}$ with elements of the form $U=\left(\begin{array}{c}u_{1} \\ u\end{array}\right)$, where $u_{1} \in \mathbb{C}$ and $u \in \mathcal{H}_{1}$, and inner product

$$
\langle U, V\rangle_{B}=\langle u, v\rangle+u_{1}\left\langle\varphi_{1}, v\right\rangle+v_{1}^{*}\left\langle u, \varphi_{1}\right\rangle+\beta_{11} v_{1}^{*} u_{1} .
$$

The self-adjoint realization of the singular perturbation in the $B$-model is the selfadjoint operator in the representation of the function $-\left(Q_{B}(z)+\cot \theta_{B}\right)^{-1}$, and 
this operator is the closure in $\widehat{\mathcal{H}}_{B}$ of the operator $B_{\theta_{B}}$ in $\mathcal{H}_{B}$. The operator $B_{\theta_{B}}$ is the restriction of the operator

$$
B_{\max }\left(\begin{array}{c}
u_{1} \\
u_{r}+u_{2} \varphi_{2}
\end{array}\right)=\left(\begin{array}{c}
u_{2}-a_{1} u_{1} \\
L u_{r}-a_{2} u_{2} \varphi_{2}
\end{array}\right), \quad u_{r} \in \mathcal{H}_{3}, u_{1}, u_{2} \in \mathbb{C}
$$

determined by the self-adjoint "boundary" condition

$$
\left\langle u_{r}, \varphi\right\rangle+\beta_{1} u_{2}=-\cot \theta_{B} u_{1} .
$$

The correspondence between the $A$ - and $B$-models can be seen from the relation

$$
Q_{B}(z)+\cot \theta_{B}=\left(z+a_{1}\right)\left(Q_{A}(z)+\cot \theta_{A}\right)
$$

and it implies the correspondence between parameters

$$
\beta_{1}=\cot \theta_{A}, \quad \cot \theta_{B}=-\alpha .
$$

6.3. The case $n=4$

Now $\varphi \in \mathcal{H}_{-4} \backslash \mathcal{H}_{-3}$ and there are three normalization points $a_{1}, a_{2}, a_{3}>0$. Besides (6.1) we set

$$
b_{3}(z)=\left(z+a_{1}\right)\left(z+a_{2}\right)\left(z+a_{3}\right), \quad \varphi_{3}=\frac{1}{b_{3}(L)} \varphi .
$$

The $A$-model. The function $Q_{A}(z)+\cot \theta_{A}$ is of the form

$$
Q_{A}(z)+\cot \theta_{A}=\left\langle\frac{z+a_{3}}{L-z} \varphi_{3}, \varphi\right\rangle-\frac{\alpha_{1}+\alpha_{2}\left(z+a_{1}\right)}{\left(z+a_{1}\right)\left(z+a_{2}\right)}+\cot \theta_{A},
$$

where $\alpha_{1}, \alpha_{2} \in \mathbb{R}$ and $\theta_{A} \in[0, \pi)$. The space of the realization is

$$
\mathcal{H}_{A}=\mathbb{C}^{2} \oplus \mathcal{H}_{2}
$$

with Gram operator

$$
G_{A}=\Gamma_{\alpha} \oplus I_{\mathcal{H}_{2}}, \quad \Gamma_{\alpha}=\left(\begin{array}{cc}
\left(a_{1}-a_{2}\right) \alpha_{1} & \alpha_{1} \\
\alpha_{1} & \alpha_{2}
\end{array}\right) .
$$

$G_{A}$ is non-degenerate only if

$$
\operatorname{det} \Gamma_{\alpha}=\left(a_{1}-a_{2}\right) \alpha_{1} \alpha_{2}-\alpha_{1}^{2} \neq 0 .
$$

The last condition is a restriction on the admissible parameters in the $A$-model. As to the signature of the space $\mathcal{H}_{A}$ :

a) $\mathcal{H}_{A}$ is a Hilbert space if $a_{1} \neq a_{2}$ and

$$
\frac{\alpha_{1}}{a_{2}-a_{1}}>0, \quad \alpha_{2}+\frac{1}{a_{2}-a_{1}}>0 .
$$

b) $\mathcal{H}_{A}$ is a Pontryagin space with negative index $=1$ if either $a_{1} \neq a_{2}$ and one of the following three alternatives are satisfied

$$
\frac{\alpha_{1}}{a_{2}-a_{1}}>0, \alpha_{2}+\frac{1}{a_{2}-a_{1}}<0 \quad \text { or } \quad \frac{\alpha_{1}}{a_{2}-a_{1}}<0, \alpha_{2}+\frac{1}{a_{2}-a_{1}}>0,
$$

or $a_{1}=a_{2}$ and $\alpha_{1} \neq 0$. 
c) $\mathcal{H}_{A}$ is a Pontryagin space with negative index 2 if $a_{1} \neq a_{2}$ and

$$
\frac{\alpha_{1}}{a_{2}-a_{1}}<0, \quad \alpha_{2}+\frac{1}{a_{2}-a_{1}}<0 .
$$

The self-adjoint realization of the singular perturbation in the $A$-model now is the operator $A_{\theta_{A}}$ in $\mathcal{H}_{A}$ defined as the restriction of the operator

$$
A_{\max }\left(\begin{array}{c}
u_{1} \\
u_{2} \\
u_{r}+u_{3} \varphi_{3}
\end{array}\right)=\left(\begin{array}{c}
u_{2}-a_{1} u_{1} \\
u_{3}-a_{2} u_{2} \\
L u_{r}-a_{3} u_{3} \varphi_{3}
\end{array}\right), \quad u_{r} \in \mathcal{H}_{4}, u_{1}, u_{2}, u_{3} \in \mathbb{C},
$$

by the self-adjoint "boundary" condition

$$
\left\langle u_{r}, \varphi\right\rangle-\alpha_{1} u_{1}-\alpha_{2} u_{2}=-\cot \theta_{A} u_{3} .
$$

The $B$-model. The function $Q_{B}(z)+\cot \theta_{B}$ has the form

$$
Q_{B}(z)+\cot \theta_{B}=b_{3}(z)\left\langle\frac{1}{L-z} \varphi_{3}, \varphi\right\rangle+\beta_{2}\left(z+a_{1}\right)\left(z+a_{2}\right)+\beta_{1}\left(z+a_{1}\right)+\cot \theta_{B},
$$

where $\beta_{1}, \beta_{2} \in \mathbb{R}$ and $\theta_{B} \in[0, \pi)$. The space of the realization is

$$
\widehat{\mathcal{H}}_{B}=\mathbb{C} \oplus \mathbb{C} \oplus \mathcal{H}
$$

with Gram operator

$$
\begin{gathered}
G_{B}=\left(\begin{array}{cc}
0 & 1 \\
1 & \beta_{11}
\end{array}\right) \oplus I_{\mathcal{H}}, \\
\beta_{11}=\beta_{1}+\left(a_{2}-a_{1}\right) \beta_{2}+\left(a_{3}-a_{1}\right)\left(a_{2}-a_{1}\right)\left\langle\varphi_{3}, \varphi_{1}\right\rangle
\end{gathered}
$$

and $\widehat{\mathcal{H}}_{B}$ is a Pontryagin space with negative index $=1$.

The space $\widehat{\mathcal{H}}_{B}$ is the completion of the pre-Pontryagin space $\mathcal{H}_{B}$ which is the linear space of the elements $U=\left(\begin{array}{l}\vec{u} \\ u\end{array}\right)$, where $\vec{u}=\left(\begin{array}{l}u_{1} \\ u_{2}\end{array}\right) \in \mathbb{C}^{2}$ and $u \in \mathcal{H}_{2}$, and endowed with the inner product

$$
\langle U, V\rangle_{B}=\langle u, v\rangle+\sum_{j=1}^{2}\left(u_{j}\left\langle\varphi_{j}, v\right\rangle+v_{j}^{*}\left\langle u, \varphi_{j}\right\rangle\right)+\sum_{j=1}^{2} \sum_{k=1}^{2} \beta_{j k} v_{j}^{*} u_{k},
$$

where

$$
\beta_{12}=\beta_{21}=\beta_{2}+\left(a_{3}-a_{1}\right)\left\langle\varphi_{3}, \varphi_{1}\right\rangle, \quad \beta_{22}=\left\langle\varphi_{2}, \varphi_{2}\right\rangle .
$$

The self-adjoint realization of the singular perturbation in the $B$-model is the selfadjoint operator in the representation of the function $-\left(Q_{B}(z)+\cot \theta_{B}\right)^{-1}$, and this is the closure in $\widehat{\mathcal{H}}_{B}$ of the operator $B_{\theta_{B}}$ in $\mathcal{H}_{B}$. The operator $B_{\theta_{B}}$ is the restriction of the operator

$$
B_{\max }\left(\begin{array}{c}
u_{1} \\
u_{2} \\
u_{r}+u_{3} \varphi_{3}
\end{array}\right)=\left(\begin{array}{c}
u_{2}-a_{1} u_{1} \\
u_{3}-a_{2} u_{2} \\
L u_{r}-a_{3} u_{3} \varphi_{3}
\end{array}\right), \quad u_{r} \in \mathcal{H}_{4}, u_{1}, u_{2}, u_{3} \in \mathbb{C},
$$

by the self-adjoint "boundary" condition

$$
\left\langle u_{r}, \varphi\right\rangle+\beta_{1} u_{2}+\beta_{2} u_{3}=-\cot \theta_{B} u_{1} .
$$


The correspondence between the $A$ - and $B$-models is given via the relations

$$
Q_{B}(z)+\cot \theta_{B}=\left(z+a_{1}\right)\left(z+a_{2}\right)\left(Q_{A}(z)+\cot \theta_{A}\right)
$$

which imply that

$$
\beta_{1}=-\alpha_{2}, \beta_{2}=\cot \theta_{A}, \cot \theta_{B}=-\alpha_{1}
$$

\section{References}

[1] N.I. Achieser and I.M. Glasmann, Theorie der linearen Operatoren im Hilbertraum, Akademie Verlag, Berlin, 1981.

[2] S. Albeverio and P. Kurasov, Rank one perturbations, approximations, and selfadjoint extensions, J. Funct. Anal. 148, 1997, 152-169.

[3] S. Albeverio and P. Kurasov, Singular perturbations of differential operators, Cambridge Univ. Press, 2000 (London Mathematical Society Lecture Notes 271).

[4] T.Ya. Azizov and I.S. Iokhvidov, Foundations of the theory of linear operators in spaces with an indefinite metric, Nauka, Moscow, 1986 (Russian); English translation: Linear operators in spaces with an indefinite metric, Wiley, New York, 1989.

[5] Yu.M. Berezanskii, Expansions in eigenfunctions of self-adjoint operators, Transl. Amer. Math. Soc. 17, Providence, Rhode Island, 1968.

[6] F.A. Berezin, On the Lee model, Matem. Sborn. 60, 1963, 425-453 (Russian).

[7] F.A. Berezin and L.D. Faddeev, Remark on the Schrödinger equation with singular potential, Dokl. Akad. Nauk SSSR 137, 1961, 1011-1014.

[8] J. Bognar, Indefinite inner product spaces, Springer-Verlag, Berlin, 1974.

[9] N. Bogolubuv, D. Shirkov, Introduction to theory of quantized fields, Interscience Publishers, New York, 1959.

[10] W. Caspers, On point interactions, Thesis, Technical University Delft, 1992.

[11] A. Dijksma and H. Langer, Operator theory and ordinary differential operators, Lecture Series 2 in: Albrecht Böttcher et al., Lectures on Operator theory and its applications, Fields Institute Monographs, Amer. Math. Soc., Providence RI, 1995, 73-139.

[12] A. Dijksma, H. Langer, A. Luger, and Yu. Shondin, Minimal realizations of scalar generalized Nevanlinna functions related to their basic factorization, Operator Theory: Adv., Appl., vol. 154, Birkhäuser Verlag, Basel, 2004, 69-90.

[13] A. Dijksma, H. Langer, Yu. Shondin, and C. Zeinstra, Self-adjoint operators with inner singularities and Pontryagin spaces, Operator Theory: Adv. Appl., vol. 118, Birkhäuser Verlag, Basel, 2000, 105-175.

[14] A. Dijksma and Yu. Shondin, Singular point-like perturbations of the Bessel operator in a Pontryagin space, J. Diff. Equations 164, 2000, 49-91.

[15] A. Dijksma and Yu. Shondin, Singular point-like perturbation of the Laguerre operator, Operator Theory: Adv., Appl., vol 132, Birkhäuser Verlag, Basel, 2002, 141-181.

[16] A. Dijksma and H.S.V. de Snoo, Symmetric and self-adjoint relations in Krein spaces I, Operator Theory: Adv. Appl., vol. 24, Birkhäuser Verlag, Basel, 1987, 145-166.

[17] J.F. van Diejen and A. Tip, Scattering from generalized point interaction using selfadjoint extensions in Pontryagin spaces, J. Math. Phys. 32(3), 1991, 630-641. 
[18] I.S. Iokhvidov, M.G. Krein, and H. Langer, Introduction to the spectral theory of operators in spaces with an indefinite metric, Mathematical Research, vol. 9, AcademieVerlag, Berlin, 1982.

[19] A.A. Kiselev and I.Yu. Popov, Higher moments in the model of zero-width slits, Teor. Mat. Fiz. 89(1), 1991, 11-17 (Russian); English translation: Theor. Math. Phys. 89, 1991, 1019-1024.

[20] A. Kiselev and I. Popov, An indefinite metric and scattering by regions with a small aperture, Mat. Zametki 58, 1995, 837-850, 959.

[21] M.G. Krein and H. Langer, Über die Q-Funktion eines $\pi$-hermiteschen Operators in Raume $\Pi_{\kappa}$, Acta Sci. Math. (Szeged) 34, 1973, 191-230.

[22] M.G. Krein and H. Langer, Über einige Fortzetzungsprobleme, die eng mit der Theorie hermitescher Operatoren im Raume $\Pi_{\kappa}$ zusammenhängen. I. Einige Funktionenklassen und ihre Darstellungen, Math. Nachr. 77, 1977, 187-236.

[23] H. Langer, A characterization of generalized zeros of negative type of functions of the class $N_{\kappa}$, Operator Theory: Adv. Appl., vol. 17, Birkhäuser Verlag, Basel, 1986, 201-212.

[24] P. Kurasov, $\mathcal{H}_{-n}$-perturbations of self-adjoint operators and Krein's resolvent formula, Integr. Eq. Oper. Theory 45, 2003, 437-460.

[25] P. Kurasov, Singular and supersingular perturbations: Hilbert space methods, Spectral theory of Schrdinger operators, 185-216, Contemp. Math., 340, Amer. Math. Soc., Providence, RI, 2004.

[26] P. Kurasov and Yu. Pavlov, On field theory methods in singular perturbation theory, Lett. Math. Phys. 64 (2003), no. 2, 171-184.

[27] P. Kurasov and K. Watanabe, On rank one $\mathcal{H}_{-3}$-perturbations of positive self-adjoint operators, Stochastic processes, physics and geometry: new interplays, II (Leipzig, 1999), 413-422, CMS Conf. Proc., 29, Amer. Math. Soc., Providence, RI, 2000.

[28] P. Kurasov and K. Watanabe, On $\mathcal{H}_{-4}$-perturbations of self-adjoint operators, Operator Theory: Adv. Appl., vol. 126, Birkhäuser Verlag, Basel, 2001, 179-196.

[29] B. Pavlov, The theory of extensions and explicitly solvable models, Uspekhi Mat. Nauk 42, 1987, 99-131.

[30] I.Yu. Popov, Helmholtz resonator and operator extension theory in spaces with indefinite metrics, Matem. Sb. 183 3, 1992, 3-37 (Russian); English translation: Russian Acad. Sci. Sb Math. 75 2, 1993, 285-315.

[31] Yu.G. Shondin, Quantum-mechanical models in $\mathbf{R}^{n}$ associated with extension of the energy operator in a Pontryagin space, Teor. Mat. Fiz. 74, 1988, 331-344 (Russian); English translation: Theor. Math. Phys. 74, 1988, 220-230.

[32] Yu.G. Shondin, Perturbation of differential operators on high-codimensional manifold and the extension theory for symmetric linear relations in an indefinite metric space, Teor. Mat. Fiz. 92(3), 1992, 466-472 (Russian); English translation: Theor. Math. Phys. 92, 1992, 1032-1037.

[33] B. Simon, Spectral analysis of rank one perturbations and applications, in: Mathematical quantum theory. II. Schrödinger operators (Vancouver, BC, 1993), 109-149, CRM Proc. Lecture Notes 8, AMS, Providence, RI, 1995. 


\author{
A. Dijksma \\ Department of Mathematics \\ University of Groningen \\ P.O. Box 800 \\ 9700 AV Groningen \\ The Netherlands \\ e-mail: dijksma@math.rug.nl \\ P. Kurasov \\ Department of mathematics \\ Lund Institute of Technology \\ P.O. Box 118 \\ 22100 Lund \\ Sweden \\ e-mail: kurasov@maths.1th.se \\ Yu. Shondin \\ Department of Theoretical Physics \\ Pedagogical State University \\ Str. Uly'anova 1, GSP 37 \\ Nizhny Novgorod 603950 \\ Russia \\ e-mail: shondin@shmath.nnov.ru
}

Submitted: November 15, 2003

Revised: February 15, 2005

\title{
(D) To access this journal online: \\ (20) http://www.birkhauser.ch
}

